\title{
Abundance of female-biased and paucity of male-biased somatically expressed genes on the mouse X-chromosome
}

Björn Reinius ${ }^{1,2,3^{*}}$, Martin M Johansson ${ }^{1}$, Katarzyna J Radomska ${ }^{1}$, Edward H Morrow ${ }^{4}$, Gaurav K Pandey ${ }^{5}$, Chandrasekhar Kanduri ${ }^{6}$, Rickard Sandberg ${ }^{2,3}$, Robert W Williams ${ }^{7}$ and Elena Jazin ${ }^{1}$

\begin{abstract}
Background: Empirical evaluations of sexually dimorphic expression of genes on the mammalian X-chromosome are needed to understand the evolutionary forces and the gene-regulatory mechanisms controlling this chromosome. We performed a large-scale sex-bias expression analysis of genes on the X-chromosome in six different somatic tissues from mouse.

Results: Our results show that the mouse X-chromosome is enriched with female-biased genes and depleted of male-biased genes. This suggests that feminisation as well as de-masculinisation of the X-chromosome has occurred in terms of gene expression in non-reproductive tissues. Several mechanisms may be responsible for the control of female-biased expression on chromosome $X$, and escape from $X$-inactivation is a main candidate. We confirmed escape in case of Tmem29 using RNA-FISH analysis. In addition, we identified novel female-biased non-coding transcripts located in the same female-biased cluster as the well-known coding X-inactivation escapee $K d m 5 c$, likely transcribed from the transition-region between active and silenced domains. We also found that previously known escapees only partially explained the overrepresentation of female-biased X-genes, particularly for tissue-specific female-biased genes. Therefore, the gene set we have identified contains tissue-specific escapees and/or genes controlled by other sexually skewed regulatory mechanisms. Analysis of gene age showed that evolutionarily old X-genes (>100 myr, preceding the radiation of placental mammals) are more frequently female-biased than younger genes.
\end{abstract}

Conclusion: Altogether, our results have implications for understanding both gene regulation and gene evolution of mammalian X-chromosomes, and suggest that the final result in terms of the X-gene composition (masculinisation versus feminisation) is a compromise between different evolutionary forces acting on reproductive and somatic tissues.

Keywords: X-chromosome, Sex chromosome, Somatic, Gene expression, Sexual antagonism, Sexual selection, Gender, Sex-bias, Female-bias, Male-bias, Sexual dimorphism, Dosage compensation, X-inactivation, Escape, Feminisation, Masculinisation, De-masculinisation, Microarray, Non-coding RNA, IncRNA, Tmem29, Kdm5c, Xist

\footnotetext{
* Correspondence: bjorn.reinius@ki.se

'Department of Organismal Biology, Evolutionary Biology Centre, Uppsala University, Uppsala, Sweden

'Ludwig Institute for Cancer Research, Stockholm, Sweden

Full list of author information is available at the end of the article
} 


\section{Background}

Females and males have different routes to maximize fitness and therefore the two sexes have different optimal transcriptomes. The X-chromosome is of particular interest in terms of understanding the evolution of sexual dimorphism [1]. Heterogamety in males (XY) and homogamety in females (XX) makes the X-chromosome a unique environment that can expose sexual genomic conflict. Sex-specific selection pressures can favour phenotypic divergence between the sexes [2]. When an allele has opposite effects on fitness in males and females (intralocus conflict) divergent evolution of associated traits is likely to be constrained, and sexual antagonism in allele selection can persist over long timescales [3-6]. Unlike autosomes, the mammalian $\mathrm{X}$-chromosomes have spent approximately $2 / 3$ of their evolutionary time in females, as compared to $1 / 3$ in males. Consequently, it might be predicted that the X-chromosome should be enriched with female-beneficial rather than malebeneficial alleles $[7,8]$, although this is also dependent on the dominance state of the alleles. Rice has argued that heterogamety puts $\mathrm{X}$ under direct selection in males, and alleles that confer differential fitness, particularly as recessives, will be strongly selected in males, leading to the gradual masculinisation of $\mathrm{X}$ during evolution [9].

Sequencing of the mammalian X-chromosomes and genome-wide expression profiling are now providing empirical data, shedding new light on these ideas. Indeed, sex-biased expression is at present commonly used as an indicator of previously resolved sexual antagonism at the genomic level [1]. For example, gene expression studies have revealed feminisation of the $\mathrm{X}$ in Drosophila melanogaster [10] and Caenohabditis elegans [11]. However, results from studies of mammalian $\mathrm{X}$-chromosomes have been partly conflicting. For example, the mouse $\mathrm{X}$-chromosome has been suggested to be both feminised and masculinised for genes expressed in tissues related to reproduction [8]. Extensive sexbiased expression also occurs in non-reproductive tissues [12]. Therefore comprehensive analyses of sexually dimorphic gene expression in somatic tissues could provide further insight into the sex-biased properties of the mammalian X-chromosome.

The current model of the evolution of the XY system describes a chain of events in which the $\mathrm{X}$ and $\mathrm{Y}$ evolved from a pair of autosomes [7]. In brief, this process involves the establishment of a testis determining factor (TDF) on the proto Y-chromosome, the repression of X/Y recombination to conserve linkage between the TDF and malebeneficial genes on $\mathrm{Y}$, and the consequential erosion of $\mathrm{Y}$ which cause the need for up-regulation of X-linked genes in males to restore the X-chromosome:Autosomal ratio of critical genes $[13,14]$. Mammalian females have a double dose of all genes located on the non-recombining region of the $\mathrm{X}$ relative to males. To reach an equal expression level of X-genes between the sexes, mechanisms evolved to transcriptionally silence one of the two copies of genes encoded on the Xs in females. This is achieved by a chromosome-wide mechanism termed X-chromosome inactivation (XCI) [15]. In female cells, one $\mathrm{X}$ is transcriptionally inactive $(\mathrm{Xi})$ while the other remains active $(\mathrm{Xa})$. The selection of $\mathrm{Xi}$ and $\mathrm{Xa}$ occurs at random in each cell during early development and the silenced state of one $\mathrm{X}$ is maintained during cell division throughout adulthood. $\mathrm{XCI}$ makes general theoretical predictions of masculinisation versus feminisation of the gene content on $\mathrm{X}$ complex [16]. For example, for circulating gene products females might be effectively heterozygous while for intracellular gene products they might be effectively hemizygous chimeras. Empirical investigations of sex-biased expression of the X-chromosome are therefore essential for understanding the evolution of this chromosome. Furthermore, the silence of $\mathrm{Xi}$ is not complete. It has been known since the 1970s that some genes "escape" XCI and are expressed from both X-alleles $[17,18]$. Early investigations showed that "escapees" are located in disjoint parts of the $\mathrm{X}$-chromosome, and that escape proceeds by a mechanism that allows for considerable autonomy between different genes and regions on the X-chromosome $[19,20]$. Now, forty years later, we know many more genes that escape $\mathrm{XCI}$ in humans and mouse, but the molecular mechanisms determining escape remain unclear. It is established that escape from XCI can produce gene expression differences between males and females, and that this is one important mechanism for explaining how female and male phenotypes can be obtained from two similar genomes $[1,21]$. Escape from XCI is not the only mechanism that can produce sex-biased expression on $\mathrm{X}$. A careful and comprehensive combined analysis of gene content, escape of inactivation, imprinting as well as other allelic asymmetries and their epigenetic control mechanisms will be required in order to understand the selective forces operating on X. For this, robust identification of sex-biased genes located on the $\mathrm{X}$-chromosome is needed. Several previous studies concerned the distribution and expression of X-genes with functions in reproductive tissues, but equally extensive studies of sex-biased expression in somatic tissues are still lacking. To remedy this, we performed large-scale analyses of microarray hybridisations with male and female RNA from multiple somatic mouse tissues including kidney, liver, lung, eye, and two brain regions: the striatum and hippocampus.

\section{Results}

\section{High-resolution screening for sex-biased X-genes in} somatic tissues

Our first goal was to build a comprehensive list of genes encoded on the mouse X-chromosome with sex-biased expression in somatic tissues. To provide sufficient 

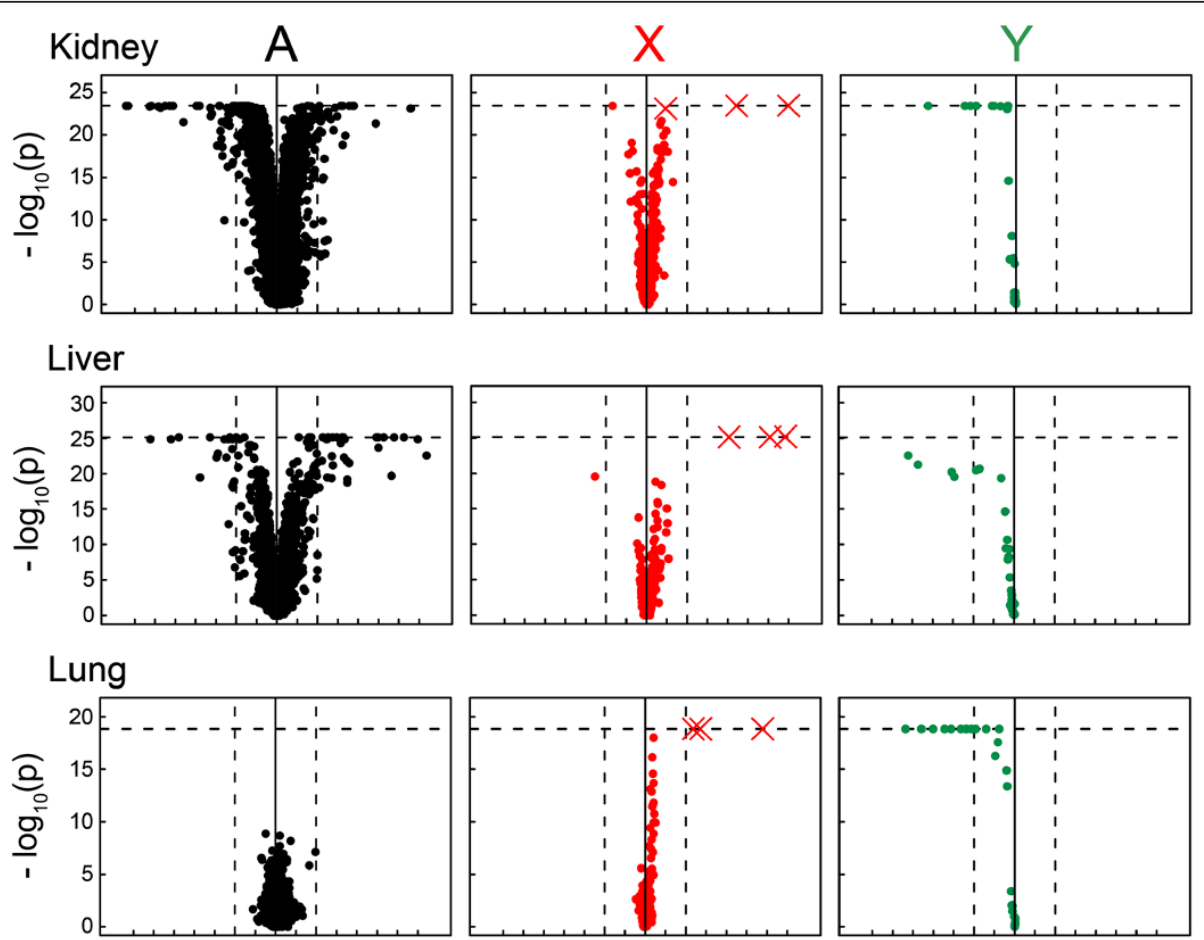

\section{Striatum}
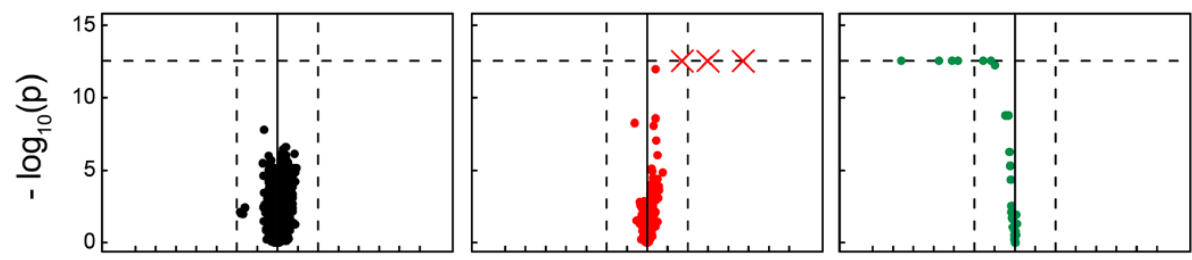

Eye
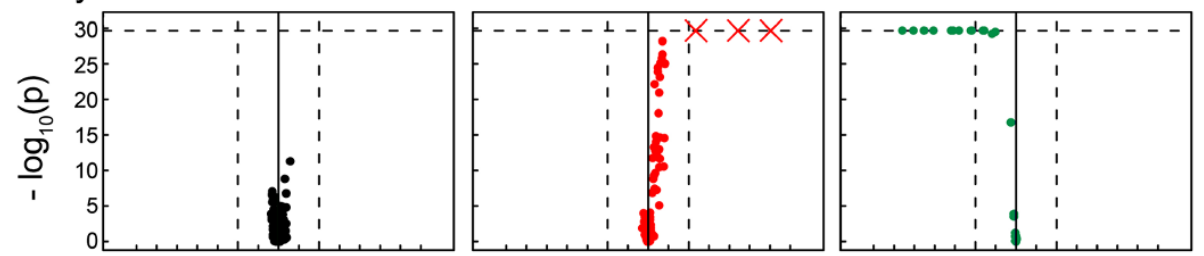

\section{Hippocampus}
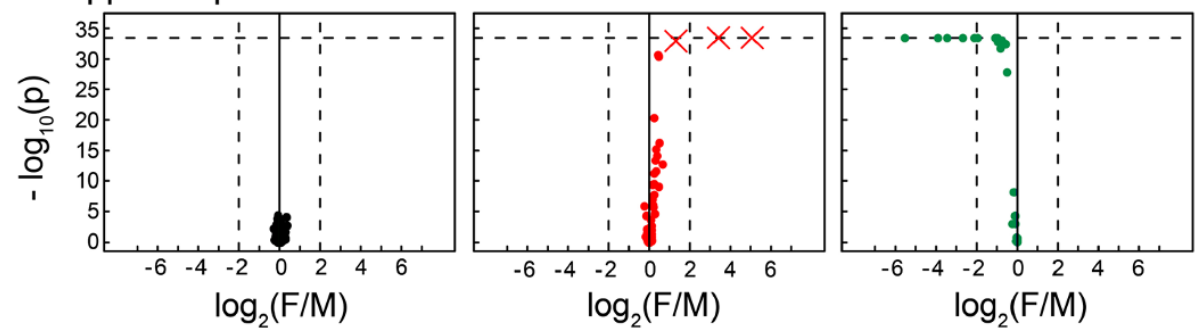

Figure 1 Overview of the microarray results. The data is separated according to chromosomal mapping: Autosomes (A), X-chromosome ( $X$ ) and $Y$-chromosome $(Y)$. On the $x$-axis: mean female/male $\log _{2}$ fold-changes; negative values represent male-bias and positive values represent female-bias. On the $y$-axis: $-\log _{10} \mathrm{p}$-values (two-sided Wilcoxon test). Each dot represents a probe and the data points marked with crosses represent probes for Xist. The horizontal dashed lines indicate the significance level at which probe intensities presented a non-overlapping distribution between the sexes; probes lying on this line show higher expression in one sex relative to the other in all possible combinations of individual comparisons. The vertical dashed lines mark the fold-change $+/-4$ (i.e. 2 on $\log _{2}$ scale), included in the figure for the sake of comparisons. 
statistical power to detect even small expression differences, we analyzed a large collection of oligonucleotide microarrays $\left(\mathrm{n}_{\text {arrays }}=728\right.$, Affymetrix Mouse 430v.2) hybridized with RNA samples obtained from six somatic tissues: kidney $\left(\mathrm{n}_{\text {arrays }}=152\right.$, with $\mathrm{n}_{\text {female arrays }}$ : $\mathrm{n}_{\text {male arrays }}$ equal to $99: 53)$, liver $(88,44: 44)$, lung $(67,33: 34)$, striatum $(46,25: 21)$, eye $(176,88: 88)$ and hippocampus (199, 101:98). To identify differentially expressed genes, we applied Wilcoxon rank-sum test (results from parametric tests are broadly similar, see Methods). The result from this analysis is graphically presented in Figure 1, including data for $\mathrm{X}, \mathrm{Y}$ and autosomal chromosomes. Table 1 gives an overview of the results obtained at the significance threshold $\mathrm{p}<0.001$. Detailed data for all individual genes and tissues are found in Additional file 1 and Additional file 2.

A first observation was that the overall percentage of sex-biased transcripts encoded on the X-chromosome was higher than the percentage of sex-biased transcripts encoded on the autosomes, and this observation was consistent in all tissues (See columns labelled "Autosomes Tot" and "X-chromosome Tot", Table 1). Therefore, it can be concluded that the X-chromosome is a chromosomal location overrepresented for sexbiased genes, in agreement with previous results [12]. Also in line with previous investigations, we observed substantial tissue differences in the number of sexbiased genes (Table 1). This was the case when considering only the X-chromosome (ranging from 25 to 287 transcripts) as well as when considering autosomes (8-7825 transcripts), while the number of Y-linked genes remained nearly constant between the tissues analysed (12-17 transcripts).
Large number of genes with small magnitude sex-biased expression on $\mathrm{X}$

We found that while sex-biased genes located on $X$ are numerous, the magnitude of their sex differences was small, and this was valid for all somatic tissues investigated. For example, while 80 autosomal transcripts showed sex differences larger than 4-fold in the kidney, not a single transcript with such a large difference was found on the $\mathrm{X}$-chromosome when Xist (X-inactive specific transcript, "Master regulator" of XCI) was excluded (Additional file 1). In the liver, 53 autosomal transcripts were sex-biased beyond 4-fold, while only a single malebiased X-linked gene (Alas2) showed differences beyond 4-fold (Xist excluded). These small differences are better illustrated in Figure 1, where only three crosses, all of them representing probes for parts of the Xist gene, resulted in female-bias greater than 4-fold. At the same time, the figure shows results for many autosomal transcripts with large sexual expression differences, particularly in the liver and kidney. Genes encoded by the Y-chromosome also resulted in large differences in all tissues as expected, since they are only expressed in males (Figure 1). The spatial distribution of sex-biased genes along the X-chromosome is illustrated in Figure 2A, where it can be observed that their distribution is widespread over the chromosome rather than concentrated on specific chromosomal segments, and does not significantly diverge from the overall gene density on the $\mathrm{X}$.

\section{Abundance of female-biased and paucity of male-biased genes on $X$}

To study the relative allocation of male- and femalebiased genes, we compared their frequencies on the

Table 1 Summary of the microarray results

\begin{tabular}{|c|c|c|c|c|c|c|c|c|c|c|c|c|c|c|c|c|}
\hline \multirow[t]{2}{*}{ Tissue } & \multirow[t]{2}{*}{ Probes } & \multicolumn{3}{|c|}{ All probes } & \multicolumn{3}{|c|}{ Autosomes } & \multicolumn{3}{|c|}{ X-chromosome } & \multicolumn{3}{|c|}{ Y-chromosome } & \multicolumn{3}{|c|}{ Location N/A } \\
\hline & & Tot & $F$ & $M$ & Tot & $F$ & $M$ & Tot & $F$ & $M$ & Tot & $F$ & $M$ & Tot & $F$ & $M$ \\
\hline \multirow[t]{2}{*}{ Kidney } & \# & 8153 & 4360 & 3793 & 7825 & 4157 & 3668 & 287 & 196 & 91 & 15 & 0 & 15 & 26 & 7 & 19 \\
\hline & $\%$ & 18.1 & 9.67 & 8.41 & 18.2 & 9.67 & 8.54 & 20.1 & 13.7 & 6.38 & 45.5 & 0 & 45.5 & 3.87 & 1.04 & 2.83 \\
\hline \multirow[t]{2}{*}{ Liver } & \# & 5193 & 2420 & 2773 & 4961 & 2296 & 2665 & 176 & 111 & 65 & 15 & 0 & 15 & 41 & 13 & 28 \\
\hline & $\%$ & 11.5 & 5.37 & 6.15 & 11.5 & 5.34 & 6.20 & 12.3 & 7.78 & 4.56 & 45.5 & 0 & 45.5 & 6.11 & 1.94 & 4.17 \\
\hline \multirow[t]{2}{*}{ Lung } & \# & 563 & 304 & 259 & 491 & 259 & 232 & 48 & 42 & 6 & 16 & 0 & 16 & 8 & 3 & 5 \\
\hline & $\%$ & 1.25 & 0.67 & 0.57 & 1.14 & 0.60 & 0.54 & 3.36 & 2.94 & 0.42 & 48.5 & 0 & 48.5 & 1.19 & 0.45 & 0.75 \\
\hline \multirow[t]{2}{*}{ Striatum } & $\#$ & 747 & 541 & 206 & 690 & 497 & 193 & 38 & 37 & 1 & 12 & 0 & 12 & 7 & 7 & 0 \\
\hline & $\%$ & 1.66 & 1.20 & 0.46 & 1.61 & 1.16 & 0.45 & 2.66 & 2.59 & 0.07 & 36.4 & 0 & 36.4 & 1.04 & 1.04 & 0 \\
\hline \multirow[t]{2}{*}{ Eye } & $\#$ & 106 & 65 & 41 & 49 & 28 & 21 & 38 & 36 & 2 & 17 & 0 & 17 & 2 & 1 & 1 \\
\hline & $\%$ & 0.24 & 0.14 & 0.09 & 0.11 & 0.07 & 0.05 & 2.66 & 2.52 & 0.14 & 51.5 & 0 & 51.5 & 0.30 & 0.15 & 0.15 \\
\hline \multirow[t]{2}{*}{ Hippocampus } & $\#$ & 50 & 26 & 24 & 8 & 2 & 6 & 25 & 23 & 2 & 16 & 0 & 16 & 1 & 1 & 0 \\
\hline & $\%$ & 0.11 & 0.06 & 0.05 & 0.02 & 0.00 & 0.01 & 1.75 & 1.61 & 0.14 & 48.5 & 0 & 48.5 & 0.15 & 0.15 & 0 \\
\hline
\end{tabular}

The table presents the number (\#) and the percentage (\%) of significantly sex-biased assayed probes ( $<<0.001$, two-sided Wilcoxon test) separated by chromosomal location. Abbreviations: Tot Total, $F$ female-biased, $M$ male-biased, $N / A$ chromosomal location not available. Complete data for all individual probes is available in Additional file 1 and Additional file 2. 


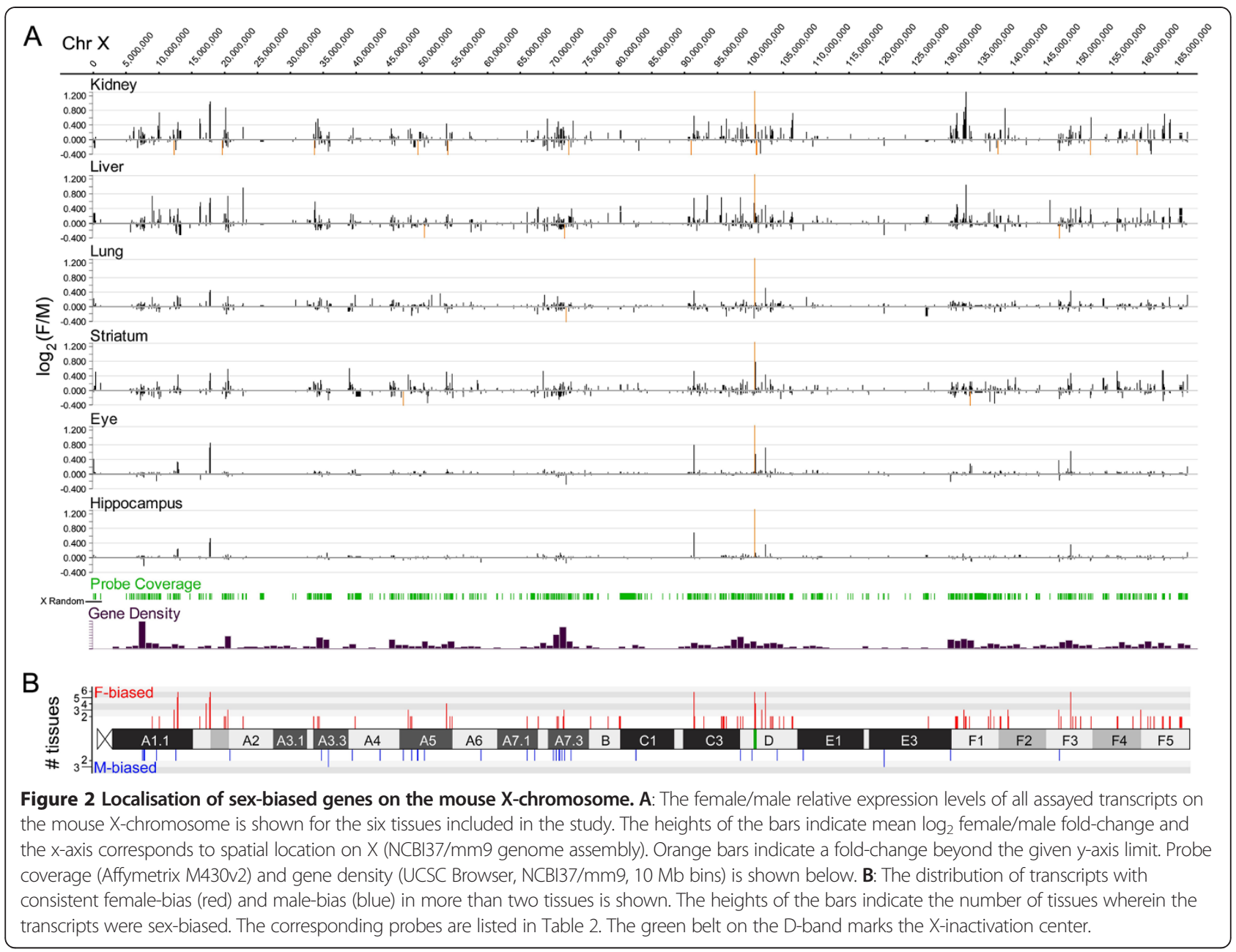

$\mathrm{X}$-chromosome. We found significantly more transcripts with female-bias than with male-bias in all six somatic tissues examined (Figure 3A, comparison $\mathrm{X}_{\mathrm{F}}$ vs. $\mathrm{X}_{\mathrm{M}}$, $\mathrm{p}<0.001)$. Next, we compared the $\mathrm{X}$ with autosomes, and found female-biased genes to be more abundant on the $\mathrm{X}$ in all tissues (Figure $3 \mathrm{~A}$, comparison $\mathrm{X}_{\mathrm{F}} v s . \mathrm{A}_{\mathrm{F}} \mathrm{p}<0.001$ ). More remarkable, in the most sexually dimorphic somatic tissues analysed: the kidney and liver, we found a significant depletion of male-biased genes on the $\mathrm{X}$ relative to the frequencies on autosomes (Figure 3A, comparison $\mathrm{X}_{\mathrm{M}}$ vs. $\mathrm{A}_{\mathrm{M}}$, kidney: $25 \%$ relative depletion, $\mathrm{p}=0.0032$ liver: $27 \%$ relative depletion, $\mathrm{p}=0.0099)$. These observations provide the first evidence of de-masculinisation of a mammalian X-chromosome in terms of X-gene expression in somatic tissues. The overall frequencies of male-biased genes in the remaining four tissues were too low to allow similar meaningful comparisons between $\mathrm{X}$ and autosomes in those tissues.

To evaluate whether biallelic expression of genes previously known to escape XCI could explain the excess of female-biased transcripts observed on $\mathrm{X}$, we compared the frequencies of male- and female-biased X-genes
(Figure 3B, left panel) with the frequencies of sex-biased genes excluding previously known mouse escapees (Figure 3B, right panel). As expected, the exclusion of escapees reduced the female to male imbalance on the $\mathrm{X}$, however, a substantial excess of female-biased genes remained. This suggests the existence of novel tissuespecific escapees in our gene sets, and/or other sexskewed mechanisms exist that regulate the expression of the remaining female-biased genes.

\section{Small female-biased gene clusters on $\mathrm{X}$}

We have previously identified small female-biased gene clusters on the mouse X-chromosome, including femalebiased long non-coding RNAs (lncRNAs) and protein coding genes that escape XCI, raising the possibility that such lncRNAs might be involved in the epigenetic regulation of escape domains [22]. Using the more extensive datasets included in the current study, we searched for additional female-biased clusters or additional genes in previously found clusters. To do this, we performed a second analysis in which the significance cut-off was relaxed from $\mathrm{p}<0.001$ to $\mathrm{p}<0.01$, increasing the sensitivity to detect sex-biased 

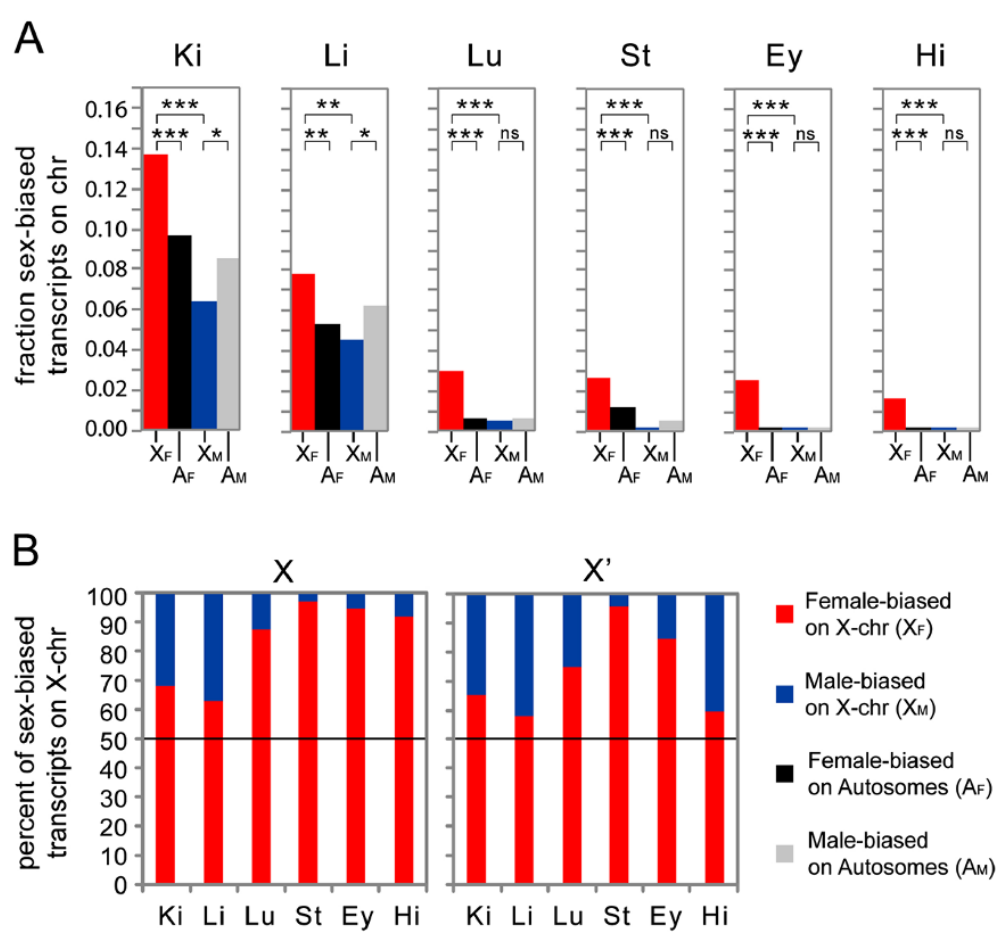

Figure 3 Abundance of female-biased and paucity of male-biased genes on X. A: Comparisons of the fraction of female-biased and malebiased transcripts within the X-chromosome and between the X-chromosome and autosomes in six somatic tissues: kidney (Ki), liver (Li), lung (Lu), striatum (St), eye (Ey) and hippocampus (Hi). P-values for differences in frequencies; ns: not significant, ${ }^{*}: p<0.01,{ }^{* *}: p<0.001,{ }^{* * *}: p<0.0001$ (two-sided Fisher's exact test). B: The relative overrepresentation of female-biased as compared to male-biased transcripts on the mouse $X$ chromosome is illustrated $(\mathbf{X})$. Overrepresentation of female-biased transcripts on $\mathbf{X}$ mostly remained after exclusion of all known $\mathrm{XCl}$ escapee genes $\left(\mathbf{X}^{\prime}\right)$. The data presented in $\mathbf{A}$ and $\mathbf{B}$ corresponds to probes selected at $\mathrm{p}<0.001$ (two-sided Wilcoxon test).

genes. At the same time, we only selected genes with consistent bias in more than one tissue, decreasing the expected number of false positives. The result from this analysis is shown in Table 2, containing 94 genes (and 8 additional non-annotated intronic sequences) with female-bias, and only 33 genes (and 2 intronic sequences) with male-bias. The spatial distribution of these genes on the X-chromosome is shown in Figure 2B. Female-biased genes located in close proximity to each other (defined as less than $50 \mathrm{~kb}$ ) are marked " $a-f$ " on the right side of Table 2. The table then shows 6 clusters, and each of them, with the exception of cluster " $e$ ", containing an IncRNA. One of the female-biased clusters " $f$ " deserves special attention because it includes several novel noncoding genes. In addition to the known escapees $K d m 5 \mathrm{c}$ (coding) and D930009K15Rik (non-coding), three additional female-biased 3'-located non-coding genes were herein identified in this cluster, including 2810454L23Rik, 1441020_at (intergenic) and 2900056M20Rik.

\section{Candidate genes for tissue-specific escape from X-inactivation}

We sought to investigate whether any of the newly identified female-biased genes might escape XCI. Candidates for this analysis were selected from Table 2, since these genes exhibit bias in at least two tissues, and therefore the evidence for possible escape from inactivation is stronger than for genes biased only in one tissue. Indeed, every gene in Table 2 that were female-biased consistently in all tissue analysed, have been previously recognised as escapees [22-25]. New selected candidates might escape $\mathrm{XCI}$ in a tissue-specific manner. As a first step, to identify novel escapee genes, we here investigated possible biallelic expression of five candidate genes in primary female mouse embryonic fibroblasts derived from skin. We performed RNA-FISH using a probe for Xist RNA in parallel with a probe for either one of the five candidate genes $\left(4732460102 R i k / R b m x^{24 / 25}, R_{b m} 4^{70^{*}}\right.$, Tmem $164^{73}$, Tmem $29^{74}$ and Ctps $2^{87}$; superscripted numbers corresponding to Table 2) and a positive control for escape: $K d m 5 c^{76} . K d m 5 c$ showed biallelic signals in $25 \%$ of the cells (13 of 52 counted, Figure 4 ). One of the candidates, Tmem29, was confirmed to partially escape XCI, showing biallelic signals in $12 \%$ of the cells (10 of 82 counted, Figure 4). None of the other candidates indicated biallelic expression in any of the examined cells, suggesting that they are $\mathrm{X}$-inactivated in mouse embryonic skin fibroblasts. The possibility 
Table 2 Genes with sex-bias in multiple tissues

\begin{tabular}{|c|c|c|c|c|c|c|c|c|c|c|c|}
\hline \multicolumn{12}{|c|}{ A. Female-biased genes } \\
\hline ProbeSet ID & Start align & Stop align & Ki & Li & Lu & St & Ey & $\mathrm{Hi}$ & Gene symbol & & \\
\hline 1426269_at & Rnd 10740 & Rnd 39163 & ns & 1.15 & 1.16 & ns & 1.31 & ns & Vamp7 & 1 & \\
\hline 1452007_at & Rnd 10740 & Rnd 39163 & ns & 1.21 & ns & ns & 1.32 & ns & Vamp7 & 1 & \\
\hline 1436778_at & 9012379 & 9015421 & ns & 1.65 & 1.19 & ns & ns & ns & $C y b b$ & 2 & \\
\hline 1436779_at & 9012379 & 9015421 & ns & 1.25 & 1.16 & ns & ns & ns & $C y b b$ & 2 & \\
\hline 1448737_at & 10062241 & 10173728 & 1.66 & 1.29 & ns & ns & ns & ns & Tspan7 & 3 & \\
\hline 1433867_at & 12232009 & 12232793 & 1.08 & 1.14 & ns & ns & 1.05 & ns & $1810030007 R_{i k}^{*}$ & 4 & \\
\hline 1416467_at & 12858147 & 12870179 & 1.21 & 1.18 & 1.22 & ns & 1.26 & 1.16 & $D d \times 3 x^{*}$ & 5 & \\
\hline 1423042_at & 12868948 & 12871177 & 1.35 & 1.21 & 1.19 & 1.35 & 1.24 & 1.18 & $D d x 3 x^{*}$ & 5 & \\
\hline 1423043_s_at & 12868948 & 12871177 & 1.37 & 1.32 & 1.14 & ns & 1.21 & 1.18 & $D d x 3 x^{*}$ & 5 & \\
\hline 1428667_at & 16196827 & 16262870 & 1.47 & 1.36 & ns & ns & ns & ns & Maoa & 6 & \\
\hline 1442676_at & 16262773 & 16263384 & 1.05 & 1.14 & ns & ns & ns & ns & Maoa & 6 & \\
\hline 1424215_at & 17133689 & 17149423 & 1.14 & 1.17 & 1.10 & ns & 1.06 & ns & Fundc1 & 7 & \\
\hline 1427672_a_at & 17740544 & 17855992 & 1.94 & 1.45 & 1.32 & ns & 1.64 & 1.32 & Kdm6a* & 8 & \\
\hline 1446278_at & 17804913 & 17805592 & 1.18 & 1.18 & 1.36 & ns & 1.46 & 1.15 & - (Kdm6a intron) & $8 \mathrm{i}$ & \\
\hline 1446234_at & 17805383 & 17806066 & 1.53 & 1.32 & 1.33 & ns & 1.76 & 1.38 & - (Kdm6a intron) & $8 \mathrm{i}$ & \\
\hline 1427235_at & 17818825 & 17856644 & 2.05 & 1.60 & 1.31 & 1.38 & 1.80 & 1.43 & Kdm6a* & 8 & \\
\hline 1445198_at & 17854669 & 17855386 & 1.09 & 1.25 & 1.26 & ns & 1.49 & 1.13 & Kdm6a* & 8 & \\
\hline 1434061_at & 19976041 & 19977980 & 1.19 & 1.19 & ns & ns & ns & ns & $R p 2 h$ & 9 & \\
\hline 1454816_at & 19976041 & 19977980 & 1.11 & 1.10 & ns & ns & ns & ns & $R p 2 h$ & 9 & \\
\hline 1448852_at & 20126913 & 20139212 & 1.82 & 1.20 & ns & ns & ns & ns & Rgn & 10 & \\
\hline 1440764_at & $\wedge 20430444$ & $\wedge 20432638$ & ns & 1.18 & ns & 1.29 & ns & ns & Araf & 11 & \\
\hline 1452279_at & 20502657 & 20508661 & ns & 1.66 & 1.21 & 1.20 & ns & ns & $C f p$ & 12 & $a$ \\
\hline 1447537_at & 20508977 & 20509301 & 1.15 & ns & ns & 1.50 & ns & ns & 1500032P08Rik & 13 & $a$ \\
\hline 1416242_at & 22796396 & 22942189 & 1.08 & 1.09 & ns & ns & ns & ns & Klh/13 & 14 & \\
\hline 1448269_a_at & 22796396 & 22942189 & 1.26 & 1.94 & ns & ns & ns & ns & KIh/13 & 14 & \\
\hline 1429028_at & 33584857 & 33616555 & 1.11 & 1.18 & ns & ns & ns & ns & Dock11 & 15 & \\
\hline 1457265_at & 34148177 & 34149112 & 1.14 & ns & ns & 1.15 & ns & ns & Sfrs $17 b$ & 16 & \\
\hline 1417609_at & 34413894 & 34424221 & 1.25 & 1.16 & ns & ns & ns & ns & Ubeza & 17 & \\
\hline 1449393_at & 39855741 & 39875274 & ns & 1.05 & 1.24 & ns & ns & ns & Sh2d1a & 18 & \\
\hline 1438916_x_at & 47908927 & 47909357 & 1.06 & 1.12 & ns & 1.32 & ns & ns & 6720401G13Rik* & 19 & \\
\hline 1435744_at & 47916296 & 47922553 & 1.19 & ns & ns & 1.34 & ns & ns & 6720401G13Rik* & 19 & \\
\hline 1419033_at & 48194223 & 48246471 & 1.22 & ns & ns & 1.21 & ns & ns & 2610018G03Rik & 20 & \\
\hline 1434678_at & 48466677 & 48468296 & 1.11 & 1.14 & ns & ns & ns & ns & $M b n / 3^{\&}$ & 21 & \\
\hline
\end{tabular}


Table 2 Genes with sex-bias in multiple tissues (Continued)

\begin{tabular}{|c|c|c|c|c|c|c|c|c|c|c|c|}
\hline 1426832_at & 53752788 & 53761020 & 1.34 & 1.13 & 1.05 & 1.41 & ns & ns & $D d \times 26 b$ & 22 & \\
\hline 1454760_at & 54307208 & 54320357 & 1.14 & ns & ns & 1.15 & ns & ns & Htatsf1 & 23 & \\
\hline 1455635_at & 54630061 & 54630758 & ns & 1.09 & ns & 1.23 & ns & ns & 4732460l02Rik & 24 & $b$ \\
\hline 1426863_at & 54636524 & 54646143 & 1.15 & ns & ns & 1.37 & ns & ns & $R b m x$ & 25 & $b$ \\
\hline 1423369_at & 65931756 & 65971138 & 1.18 & 1.23 & ns & ns & ns & ns & Fmrl & 26 & \\
\hline 1451302_at & 67639051 & 67642592 & 1.18 & 1.34 & ns & ns & ns & ns & 1110012L19Rik & 27 & \\
\hline 1435228_at & 67712927 & 67713571 & 1.18 & 1.27 & ns & ns & ns & ns & BC023829 & 28 & \\
\hline 1418397_at & 70587973 & 70604418 & 1.25 & 1.06 & ns & ns & ns & ns & Zfp275 & 29 & \\
\hline 1448323_a_at & 70728980 & 70741722 & 1.30 & 1.17 & ns & ns & ns & ns & Bgn $^{*}$ & 30 & \\
\hline 1437889_x_at & 70740822 & 70741271 & 1.17 & 1.09 & ns & ns & ns & ns & Bgn* & 30 & \\
\hline 1426677_at & 71468799 & 71481154 & 1.24 & ns & ns & 1.16 & ns & ns & Flna & 31 & \\
\hline 1448354_at & 71654824 & 71674529 & 1.08 & 1.09 & 1.07 & ns & ns & ns & G6pdx & 32 & \\
\hline 1455724_at & 75694953 & 75696134 & 1.16 & 1.07 & ns & ns & ns & ns & Prrg1 & 33 & \\
\hline 1420514_at & 78316031 & 78343314 & 1.13 & 1.21 & ns & ns & ns & ns & Tmem47 & 34 & \\
\hline 1417307_at & 80194208 & 82450389 & 1.20 & 1.24 & ns & ns & ns & ns & Dmd & 35 & \\
\hline 1448665_at & 80194208 & 82450389 & 1.20 & 1.39 & ns & ns & ns & ns & Dmd & 35 & \\
\hline 1423744_x_at & 91434045 & 91435976 & 1.53 & 1.37 & 1.34 & 1.38 & 1.41 & 1.37 & Eif2s $3 x^{*}$ & 36 & \\
\hline 1451090_a_at & 91434045 & 91435976 & 1.55 & 1.45 & 1.32 & 1.43 & 1.39 & 1.39 & Eif2s $3 x^{*}$ & 36 & \\
\hline 1421895_at & 91435260 & 91457988 & 1.48 & 1.60 & 1.34 & ns & 1.71 & 1.58 & Eif2s $3 x^{*}$ & 36 & \\
\hline 1435818_at & 91522460 & 91523059 & 1.08 & 1.06 & ns & ns & ns & ns & KIh/15 & 37 & \\
\hline 1434729_at & 92834534 & 92835231 & 1.06 & 1.07 & ns & $\mathrm{ns}$ & ns & ns & Zc4h2 & 38 & \\
\hline 1437064_at & 95514880 & 95516618 & 1.22 & 1.56 & ns & ns & ns & ns & $A r$ & 39 & \\
\hline 1455647_at & 95517026 & 95518558 & 1.29 & 1.62 & ns & ns & ns & ns & $A r$ & 39 & \\
\hline 1419108_at & 95752847 & 96086120 & 1.06 & ns & ns & 1.10 & ns & ns & Ophn1 & 40 & \\
\hline 1427072_at & 96265193 & 96270067 & 1.47 & 1.22 & ns & ns & ns & ns & Stard8 & 41 & \\
\hline 1416918_at & 97963090 & 98013749 & 1.23 & 1.10 & ns & ns & ns & ns & Dlg3 & 42 & \\
\hline 1455465_at & 98394381 & 98395191 & ns & 1.15 & ns & 1.15 & ns & ns & $-(\operatorname{Sn} \times 12$ intron $)$ & i & \\
\hline 1416295_a_at & 98459719 & 98463545 & 1.21 & 1.62 & ns & ns & ns & ns & $112 \mathrm{rg}^{\&}$ & 43 & \\
\hline 1460631_at & 98878217 & 98879690 & 1.19 & 1.20 & ns & ns & ns & ns & Ogt & 44 & \\
\hline 1440522_at & 100590759 & 100591430 & ns & 2.02 & ns & 1.17 & ns & ns & Chicl & 45 & \\
\hline 1427262_at & 100655713 & 100678556 & 128 & 114 & 55 & 7.9 & 65 & 33 & Xist $^{*}$ & 46 & \\
\hline 1427263_at & 100655713 & 100678556 & 1.9 & 16.7 & 5.1 & 3.3 & 5.1 & 2.5 & Xist $^{*}$ & 47 & \\
\hline 1436936_s_at & 100677542 & 100678588 & 22 & 67 & 6.5 & 26 & 22 & 11 & Xist $^{*}$ & 47 & c \\
\hline 1442137_at & $\wedge 100689134$ & $\wedge 100701764$ & ns & ns & 1.13 & 1.07 & 1.16 & ns & $2010000103 R i k(J p x)^{*}$ & 48 & c \\
\hline 1438838_at & 100764844 & 100765429 & 1.05 & 1.12 & ns & 1.70 & 1.40 & ns & B230206F22Rik (Ftx)* & 49 & c \\
\hline
\end{tabular}


Table 2 Genes with sex-bias in multiple tissues (Continued)

\begin{tabular}{|c|c|c|c|c|c|c|c|c|c|c|c|}
\hline 1439305_at & 100772965 & 100773674 & 1.32 & 1.20 & ns & ns & 1.46 & 1.09 & $-($ Ftx intron $)$ & $49 i$ & c \\
\hline 1435822_at & 101705781 & 101707391 & 1.19 & 1.28 & ns & 1.16 & ns & ns & D830012124Rik & 50 & \\
\hline 1438893_at & 102237486 & 102238144 & ns & ns & 1.22 & ns & 1.28 & 1.06 & 5530601H04Rik* & 51 & \\
\hline 1436347_a_at & 102238820 & 102265435 & 1.06 & 1.24 & 1.41 & 1.18 & 1.63 & 1.24 & 5530601 H04Rik* & 51 & \\
\hline 1452750_at & $\wedge 102232689$ & $\wedge 102265463$ & 1.20 & 1.33 & 1.33 & 1.34 & 1.58 & 1.27 & 5530601H04Rik* & 51 & d \\
\hline 1417921_at & 102275115 & 102278930 & 1.27 & 1.40 & 1.30 & 1.16 & 1.48 & 1.15 & 2610029G23Rik* & 52 & $\bar{d}$ \\
\hline 1433537_at & 102992735 & 102993644 & 1.30 & ns & ns & 1.23 & ns & ns & Atrx & 53 & \\
\hline 1453734_at & 102993154 & 102995719 & 1.14 & 1.13 & ns & ns & ns & ns & Atrx & 53 & \\
\hline 1418774_a_at & 103222614 & 103320808 & 1.19 & 1.09 & ns & ns & ns & ns & Atp7a & 54 & \\
\hline 1436921_at & 103321257 & 103323499 & 1.21 & 1.17 & ns & ns & ns & ns & Atp7a & 54 & \\
\hline 1457753_at & 103354989 & 103355829 & ns & 1.11 & 1.14 & ns & ns & ns & T/r13 & 55 & \\
\hline 1435584_at & 104368242 & 104369000 & 1.03 & 1.07 & ns & ns & ns & ns & A630033H20Rik & 56 & \\
\hline 1453078_at & 104978092 & 104980504 & 1.11 & 1.26 & ns & ns & ns & ns & 2610002M06Rik & 57 & \\
\hline 1421871_at & 106290703 & 106357275 & 1.43 & 1.22 & ns & ns & ns & ns & Sh3bgrl & 58 & \\
\hline 1459571_at & 106306845 & 106307385 & 1.06 & 1.15 & ns & ns & ns & ns & - (Sh3bgrl intron) & $58 \mathrm{i}$ & \\
\hline 1428107_at & 106356680 & 106357810 & 1.64 & 1.14 & ns & ns & ns & ns & Sh3bgrl & 58 & \\
\hline 1442003_at & 126999308 & 127000367 & ns & 1.18 & ns & 1.17 & ns & ns & Diap2 & 59 & \\
\hline 1416807_at & 131120240 & 131122656 & 1.23 & ns & 1.06 & ns & ns & ns & Rpl36a & 60 & \\
\hline 1425914_a_at & 131252558 & 131256449 & 1.08 & 1.08 & ns & ns & ns & ns & Armcx1 & 61 & \\
\hline 1456739_x_at & 131338685 & 131338978 & 1.64 & 1.41 & ns & ns & ns & ns & Armcx2 & 62 & \\
\hline 1435829_at & 131505913 & 131506643 & 1.16 & 1.35 & ns & ns & ns & ns & Zmat1 & 63 & \\
\hline 1428512_at & 132424218 & 132425617 & 1.69 & 1.21 & ns & 1.14 & ns & ns & Bhlhb9 & 64 & \\
\hline 1428209_at & 132673583 & 132674982 & 1.84 & 1.08 & ns & ns & ns & ns & Bex4 & 65 & e \\
\hline 1418171_at & 132704620 & 132706881 & 1.37 & 2.04 & ns & ns & ns & ns & Tceals & 66 & e \\
\hline 1451230_a_at & 132779633 & 132781675 & 1.59 & 1.11 & ns & ns & ns & ns & Wbp5 & 67 & \\
\hline 1439413_x_at & 133267489 & 133267797 & 1.14 & 1.14 & ns & ns & ns & ns & Morf4/2 & 68 & \\
\hline 1418318_at & 136145158 & 136207908 & 1.08 & 1.22 & ns & ns & ns & ns & Rnf128 & 69 & \\
\hline 1449036_at & 136145158 & 136207908 & 1.10 & 1.15 & $\mathrm{~ns}$ & ns & ns & ns & Rnf128 & 69 & \\
\hline 1456027_at & 136476076 & 136477767 & 1.06 & ns & ns & 1.10 & 1.05 & ns & Rbm41 & 70 & \\
\hline 1416052_at & 136991147 & 137010679 & 1.11 & 1.30 & ns & ns & ns & ns & Prps1 & 71 & \\
\hline 1425476_at & 137909933 & 138123778 & 1.19 & 1.15 & ns & ns & ns & ns & Col4a5 & 72 & \\
\hline 1460016_at & 139118850 & 139119428 & 1.08 & 1.09 & ns & 1.22 & ns & ns & Tmem164 & 73 & \\
\hline 1441195_at & 139127416 & 139128111 & 1.05 & ns & ns & 1.22 & ns & ns & $-($ Tmem 164 intron) & $73 i$ & \\
\hline 1454741_s_at & 139269949 & 139272918 & 1.25 & 1.16 & ns & ns & ns & ns & Tmem164 & 73 & \\
\hline 1428930_at & 146843122 & 146893683 & 1.10 & ns & 1.14 & ns & 1.29 & ns & Tmem29 & 74 & \\
\hline
\end{tabular}


Table 2 Genes with sex-bias in multiple tissues (Continued)

\begin{tabular}{|c|c|c|c|c|c|c|c|c|c|c|}
\hline 1426306_a_at & 147240996 & 147247504 & ns & 1.12 & 1.08 & ns & ns & ns & Maged2 & 75 \\
\hline 1426497_at & 148667784 & 148708632 & ns & ns & 1.26 & ns & 1.25 & 1.13 & $K d m 5 c^{*}$ & 76 \\
\hline 1426498_at & 148667784 & 148708632 & ns & ns & 1.18 & ns & 1.23 & 1.07 & $K d m 5 c^{*}$ & 76 \\
\hline 1441449_at & 148679626 & 148681294 & ns & ns & 1.08 & 1.11 & 1.15 & ns & $K d m 5 c^{*}$ & 76 \\
\hline 1441450_s_at & 148679626 & 148681294 & ns & ns & 1.18 & 1.12 & 1.19 & ns & $K d m 5 c^{*}$ & 76 \\
\hline 1457930_at & 148691371 & 148692025 & 1.14 & 1.18 & 1.24 & ns & 1.47 & 1.21 & $K d m 5 c^{*}$ & 76 \\
\hline 1440123_at & 148699256 & 148700276 & 1.10 & 1.24 & 1.29 & ns & 1.51 & 1.09 & - (Kdm5c intron) & $76 \mathrm{i}$ \\
\hline 1444157_a_at & 148708371 & 148709078 & 1.09 & 1.26 & 1.28 & 1.26 & 1.46 & 1.18 & $K d m 5 c^{*}$ & 76 \\
\hline 1444158_at & 148708371 & 148709078 & 1.28 & 1.31 & 1.24 & 1.19 & 1.44 & 1.18 & $K d m 5 c^{*}$ & 76 \\
\hline 1435348_at & 148712014 & 148713640 & 1.24 & 1.32 & 1.33 & 1.38 & 1.53 & 1.28 & D930009K15Rik & 77 \\
\hline 1428499_at & 148729448 & 148730847 & 1.08 & ns & ns & 1.10 & ns & ns & 2810454L23Rik & 78 \\
\hline 1441020_at & 148742556 & 148743349 & 1.07 & 1.14 & ns & 1.29 & ns & ns & - (Intergenic) & 79 \\
\hline 1441816_at & 148749758 & 148750124 & ns & ns & 1.12 & 1.32 & ns & ns & 2900056M20Rik & 80 \\
\hline 1433992_at & 149044532 & 149046561 & 1.15 & 1.08 & ns & ns & ns & ns & Shroom2 & 81 \\
\hline 1422498_at & 149470708 & 149472081 & 1.13 & 1.29 & ns & ns & ns & ns & Mageh1 & 82 \\
\hline 1430538_at & 150158096 & 150175958 & 1.29 & 1.36 & ns & ns & ns & ns & 2210013021 Rik & 83 \\
\hline 1416167_at & 151758460 & 151773001 & 1.50 & 1.17 & ns & ns & ns & ns & $\operatorname{Prd} \times 4$ & 84 \\
\hline 1441360_at & 155711288 & 155712253 & ns & 1.09 & ns & 1.43 & ns & ns & - (Rps6ka3 intron) & $85 i$ \\
\hline 1455206_at & 155803349 & 155806175 & ns & 1.32 & ns & 1.20 & ns & ns & Rps6ka3 & 85 \\
\hline 1452358_at & 158154967 & 158217429 & 1.10 & 1.11 & ns & ns & ns & ns & Rai2 & 86 \\
\hline 1448111_at & 159340115 & 159469264 & 1.18 & 1.32 & 1.06 & ns & ns & ns & Ctps2 & 87 \\
\hline 1452657_at & 160346948 & 160371598 & 1.12 & 1.15 & ns & ns & ns & ns & Ap1s2 & 88 \\
\hline 1438953_at & 160838594 & 160838901 & 1.18 & ns & ns & 1.10 & ns & ns & Figf & 89 \\
\hline 1438954_x_at & 160838594 & 160838901 & 1.20 & ns & ns & 1.11 & ns & ns & Figf & 89 \\
\hline 1424124_at & 161374107 & 161418260 & 1.20 & 1.26 & ns & ns & ns & ns & Mospd2 & 90 \\
\hline 1423091_a_at & 162676903 & 162826964 & 1.21 & 1.12 & ns & ns & ns & ns & Gpm6b & 91 \\
\hline 1415906_at & 163645025 & 163645984 & 1.35 & 1.33 & ns & ns & ns & ns & Tmsb4x & 92 \\
\hline 1454843_at & 163784253 & 163785400 & 1.46 & 1.13 & ns & ns & ns & ns & Prps2 & 93 \\
\hline 1417704_a_at & 165233904 & 165742756 & 1.09 & 1.14 & ns & ns & ns & ns & Arhgap6 & 94 \\
\hline 1451867_x_at & 165233904 & 165742367 & 1.14 & 1.32 & ns & ns & ns & ns & Arhgap6 & 94 \\
\hline \multicolumn{11}{|c|}{ B. Male-biased genes } \\
\hline ProbeSet ID & Start align & Stop align & Ki & $\mathrm{Li}$ & Lu & $\mathrm{St}$ & Ey & $\mathrm{Hi}$ & Gene symbol & \\
\hline 1422827_x_at & 7461369 & 7471588 & 1.08 & ns & ns & 1.11 & ns & ns & S/c35a2 & 1 \\
\hline 1432533_a_at & 7462961 & 7471586 & 1.10 & 1.10 & ns & ns & ns & ns & S/c35a2 & 1 \\
\hline 1436664_a_at & 7470132 & 7471590 & 1.10 & 1.10 & ns & ns & ns & ns & S/c35a2 & 1 \\
\hline
\end{tabular}


Table 2 Genes with sex-bias in multiple tissues (Continued)

\begin{tabular}{|c|c|c|c|c|c|c|c|c|c|c|}
\hline 1425490_a_at & 7700426 & 7709678 & 1.04 & 1.11 & ns & ns & ns & ns & Wdr13 & 2 \\
\hline 1422660_at & 7719484 & 7722860 & ns & ns & ns & ns & 1.08 & 1.18 & Rbm3 & 3 \\
\hline 1457519_at & 7729990 & 7730655 & 1.08 & 1.09 & ns & ns & ns & ns & Tbcld25 & 4 \\
\hline 1438753_at & 7779325 & 7780137 & 1.07 & 1.10 & ns & ns & ns & ns & $-($ Porcn intron $)$ & $\mathrm{i}$ \\
\hline 1451939_a_at & 9616032 & 9694713 & ns & ns & 1.11 & ns & 1.15 & ns & Srpx & 5 \\
\hline 1450039_at & 12648662 & 12749154 & 1.22 & 1.18 & ns & ns & ns & ns & Usp9x & 6 \\
\hline 1431955_at & 20783706 & 20798793 & 1.05 & ns & ns & 1.07 & ns & ns & 4930453H23Rik & 7 \\
\hline 1422241_a_at & 34727595 & 34731158 & 1.08 & 1.11 & ns & ns & ns & ns & Ndufal & 8 \\
\hline 1416344_at & 35758238 & 35809556 & 1.24 & ns & 1.03 & 1.14 & ns & ns & Lamp2 & 9 \\
\hline 1439298_at & 39437302 & 39438087 & 1.09 & 1.14 & ns & ns & ns & ns & - (Xiap intron) & $\bar{i}$ \\
\hline 1449825_at & 43682183 & 43683528 & 1.05 & 1.08 & ns & ns & ns & ns & Actrt1 & 10 \\
\hline 1433652_at & 47135712 & 47137009 & ns & 1.09 & ns & 1.55 & ns & ns & Igsf1 & 11 \\
\hline 1442549_at & 48470442 & 48471160 & ns & 1.10 & 1.19 & ns & ns & ns & $M b n / 3^{\&}$ & 12 \\
\hline 1453453_at & 49321871 & 49325949 & 1.03 & 1.08 & ns & ns & ns & ns & 1700080016Rik & 13 \\
\hline 1443620_at & 49403554 & 49404019 & 1.21 & ns & 1.14 & ns & ns & ns & Gpc4 & 14 \\
\hline 1448736_a_at & 50341268 & 50374837 & 1.17 & 1.32 & ns & ns & ns & ns & Hprt & 15 \\
\hline 1435815_at & 58963393 & 58964128 & ns & 1.16 & ns & ns & ns & 1.10 & Ldoc1 & 16 \\
\hline 1434739_at & 66015028 & 66057768 & 1.07 & 1.21 & ns & ns & ns & ns & Fmrinb & 17 \\
\hline 1458014_at & 67115572 & 67116574 & ns & 1.06 & ns & 1.05 & ns & ns & Aff2 & 18 \\
\hline 1421536_at & 70070608 & 70083883 & 1.03 & 1.05 & ns & ns & ns & ns & Gabra & 19 \\
\hline 1417412_at & 70473356 & 70475147 & 1.06 & ns & ns & 1.10 & ns & ns & $F 8 a$ & 20 \\
\hline 1422711_a_at & 70901332 & 70905177 & ns & 1.14 & ns & 1.19 & ns & ns & Pnck & 21 \\
\hline 1451049_at & 70931516 & 70961761 & 1.13 & ns & ns & 1.13 & ns & ns & Bcap31 & 22 \\
\hline 1438120_x_at & 71260144 & 71260313 & 1.19 & 1.11 & ns & ns & ns & ns & Irak1 & 23 \\
\hline 1450161_at & 71669950 & 71694950 & 1.07 & 1.11 & ns & ns & ns & ns & $1 \mathrm{kbkg}$ & 24 \\
\hline 1437553_at & 72698339 & 72699357 & 1.06 & 1.07 & ns & ns & ns & ns & Brcc3 & 25 \\
\hline 1429793_at & 82514833 & 82515627 & 1.06 & 1.09 & ns & ns & ns & ns & 1600014K23Rik & 26 \\
\hline 1458481_at & 98456711 & 98459331 & 1.04 & 1.10 & ns & ns & ns & ns & $112 \mathrm{rg}^{\&}$ & 27 \\
\hline 1447725_at & 100211276 & 100211547 & 1.06 & 1.11 & ns & ns & ns & ns & C030034E14Rik & 28 \\
\hline 1437355_at & 104032421 & 104033595 & 1.04 & 1.08 & ns & ns & ns & ns & Zcchc5 & 29 \\
\hline 1422164_at & 108009755 & 108012520 & 1.06 & 1.17 & ns & ns & ns & ns & Pou3f4 & 30 \\
\hline 1444668_at & 120324027 & 120326909 & 1.13 & 1.25 & 1.15 & ns & ns & ns & Astx & 31 \\
\hline 1417979_at & 130385542 & 130400116 & 1.03 & ns & ns & ns & 1.17 & ns & Tnmd & 32 \\
\hline 1425954_a_at & 147018680 & 147022624 & 1.05 & 1.14 & ns & ns & ns & ns & Apex2 & 33 \\
\hline
\end{tabular}

The female-biased (A) and male-biased (B) genes with the same direction of sex-bias in more than one tissue are listed. The mean fold-changes for significant differences are given for each tissue. Fold-changes in Italic font denote the significance level $p<0.01$, and fold-changes in bold font denote the significance level $p<0.001$. The letters " $a-f^{\prime}$ ", to the right in the table, indicate female-biased gene clusters as described in the main text. The additional notation used in the table is as follows. i: intronic transcript. $\wedge$ : the exact probe mapping coordinates was not specified in the array annotation file, and gene start/stop locations are therefore given according to the NCBI37/mm9 genome assembly. ${ }^{8}$ : these genes showed opposing sex-bias for different probes. *: known XCI escapee gene. 


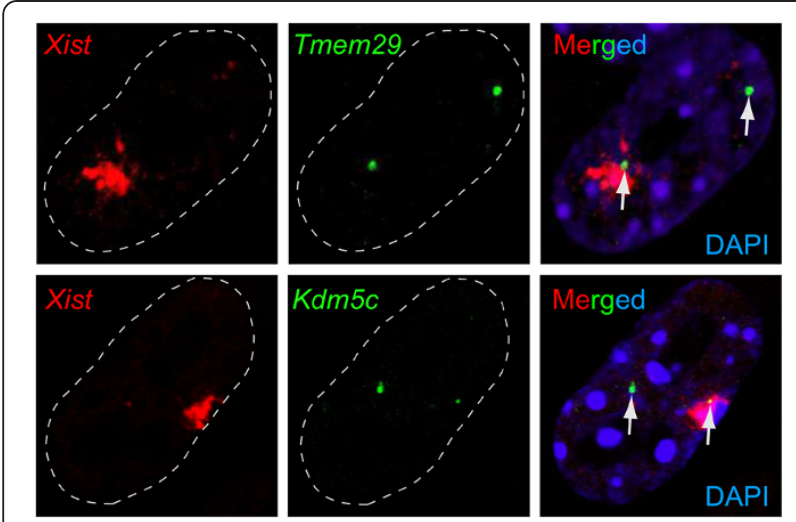

Figure 4 Confirmation of a candidate $\mathrm{XCl}$ escapee gene. The figure displays results from RNA-FISH experiments on female embryonic fibroblasts. The upper panel shows a cell with biallelic RNA signals for the candidate gene Tmem29, which escaped in 12\% of the cells (82 cells counted). The lower panel shows a cell with biallelic RNA signals for a positive control for escape, $K d m 5 c$, which escaped in $25 \%$ of the cells (52 cells counted).

remains that they might escape inactivation in other cell types.

\section{Gene ontology analysis}

To investigate whether sex-biased X-linked genes are associated with a specific functionality that is overrepresented, we performed a Gene Ontology classification $[26,27]$. To do this, we contrasted the proportion of sex-biased genes in each functional category with the proportion of the same functional category among all genes in the genome (Additional file $3 \mathrm{~A}$ ) or with all genes on the X-chromosome (Additional file $3 \mathrm{~B}$ ). These analyses revealed three groups with significant overrepresentation ( $\mathrm{p}<0.05$ after Bonferroni correction) in the genome-wide comparison, namely IPR006911: Protein of unknown function DUF634 ( $\mathrm{p}=3 \mathrm{E}-06$; overrepresented among female-biased genes in kidney), GO:0031090 Organelle membrane $(\mathrm{p}=0.009$; overrepresented among male-biased genes in kidney), and perhaps most interesting because of possible sex differences in synaptic plasticity: mmu04720: Long-term potentiation $(\mathrm{p}=0.04$; among female-biased genes in striatum).

\section{Female-biased $\mathrm{X}$-genes pre-date mammalian radiation}

It was earlier shown that genes on the $\mathrm{X}$-chromosome with female-biased function (defined by high expression in female reproductive tissues) are mainly evolutionarily old genes, while genes with male-biased function (defined by high expression in male reproductive tissues) are enriched among more recently formed genes $(<50$ myr) [28]. To investigate whether similar conclusions hold for X-genes with sex-biased expression in nonreproductive tissues, we determined the age of the $\mathrm{X}$-linked genes included in our dataset. To do this, we used gene age data obtained recently by comparative genomic analysis for the presence or absence of orthologs in a vertebrate phylogeny [28], and we calculated the proportion of female- and male-biased genes among the genes assigned to each branch in the phylogeny. We found that the proportion of genes with female-biased expression in kidney and liver was higher among evolutionarily old genes, preceding the radiation of placental mammals, than among younger genes (Figure 5). The difference in proportion of female-biased genes older than 100 myr (branch $0-5)$ versus younger genes $(<100$ myr, branch 6-11) was significant both in kidney $(\mathrm{p}=1.6 \mathrm{E}-6$, two-sided Fischer's exact test) and liver $(\mathrm{p}=1.9 \mathrm{E}-4)$. A modestly significant age-related decrease in male-biased genes was observed in kidney $(\mathrm{p}=0.032)$, while no significant difference was found in liver $(p=0.64)$. Similar analyses of the other somatic tissues included in our study were not possible due to the low number of sex-biased genes detected in those tissues.

\section{Discussion}

Skewed allocation of male- and female-biased X-genes in somatic tissues

We have used a large sample set of oligonucleotide microarrays to identify with high-resolution transcripts encoded on the X-chromosome that are expressed in a sex-biased manner in somatic tissues. We observed a skewed allocation of sex-biased somatic transcripts encoded on the X-chromosome: namely an abundance of female-biased transcripts and a paucity of male-biased transcripts. A previous large genome-wide analysis in mice, including four somatic tissues (liver, adipose, muscle and brain) also found over-representation of female-biased genes on the $X$ [12]. However, underrepresentation of male-biased genes was not reported, possibly due to the lower probe content of their arrays. Our study might thus provide the first empirical evidence of de-masculinisation of a mammalian $\mathrm{X}$-chromosome in terms of X-gene expression in somatic tissues. Our investigation of somatic tissues complements earlier analyses of sex-biased gene expression on the X-chromosome that investigated expression in reproduction-related tissues such as testis, ovary and placenta [29]. Khil et al. demonstrated that genes enriched in mouse testis are under-represented on the $\mathrm{X}$-chromosome, likely as a consequence of meiotic sex chromosome inactivation (MSCI), and at the same time, genes with high expression in ovary and placenta were shown to be enriched [29]. We here demonstrated a similarly skewed allocation of male- and female-biased $\mathrm{X}$-genes in somatic tissues. However, a crucial difference is that the observed paucity of male-biased genes in somatic tissues is unlikely to be explained by MSCI, since this process does not occur in these tissues. The 


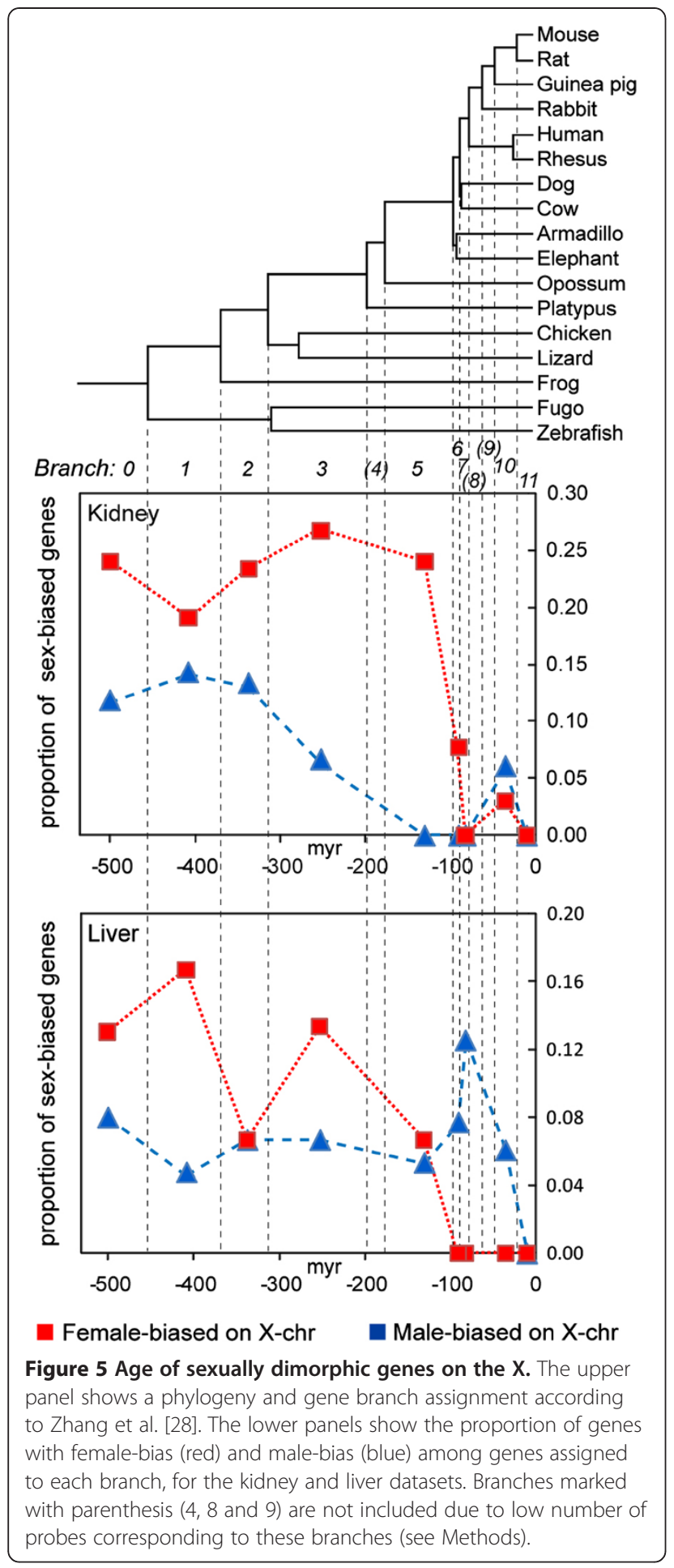

results are relevant in terms of interpreting the Rice hypothesis [9]. This hypothesis states that any recessive allele on the $\mathrm{X}$ that gives males a reproductive advantage is immediately available for positive selection since males carry a single X-chromosome. Accordingly, an excess of male-beneficial genes should accumulate on the X. This may be the case for genes specifically expressed in spermatogonia [30], but seemingly not for genes enriched in whole testis [29] or for genes expressed in somatic tissues, as indicated in our current study. Therefore, it is most plausible that different selection pressures operate on X-genes active in different tissues, and that the final result in terms of the evolution of gene composition in the $\mathrm{X}$ (masculinisation versus feminisation) is a compromise between different evolutionary forces acting on reproductive and somatic tissues, as has been hypothesised both for rodents [8] and similarly for Drosophila [31]. Our data gives support to this hypothesis.

The importance of our results can also be discussed in relationship to studies of gene composition of the sex chromosomes of other species. Indeed, the paucity of male-biased genes in both reproductive and somatic tissues of the mouse is similar to that reported in Drosophila melanogaster [10] and Caenohabditis elegans [11]. Interestingly, while the overall pattern is the same in many species, the particular genes are not. Moreover, the collections of genes biased in reproductive tissues are different from those found in somatic tissues from the same species $[32,33]$. Nonetheless, the similarity in our results from the mouse $\mathrm{X}$-chromosome and in the evolutionarily unrelated X-chromosomes in distant species such as Drosophila and Caenohabditis elegans, indicate the generality of at least some selective forces acting on X-chromosomes.

\section{Genes female-biased in multiple tissues reveal genes that escape X-inactivation}

The analysis of genes with sex-bias in more than one tissue revealed clues to the potential mechanisms controlling sex-bias of X-encoded genes as well as to whether such mechanisms operate in a ubiquitous versus tissuespecific fashion. Interestingly, while several of the genes listed in Table 2were female-biased in all six tissues, none of the male-biased X-linked transcripts were consistently biased in four or more tissues. Indeed, only two transcripts, Lamp 2 and Astx, were male-biased in more than two tissues. This was in stark contrast to the widespread expression differences of several female-biased transcripts, with 25 unique genes female-biased in more than two tissues (Table 2). These observations are in agreement with the conclusion that the mechanisms controlling female-bias versus male-bias are different. It is worth noting that all transcripts that were femalebiased in all tissues were encoded by genes known to escape XCI [22-25], namely $D d x 3 x, K d m 6 a$, Eif2s3x, Xist, 5530601H04Rik, 2610029G23Rik, Kdm5c, and D930009K15Rik. Our microarray analysis of somatic tissues, together with previous global analysis of escape from XCI performed on fibroblasts in vitro [23], indicate that most of the ubiquitously expressed coding escapee 
genes in mouse have now been identified. On the other hand, the fact that most of the genes included in Table 2were female-biased in a tissue-specific manner, might suggest that escape from XCI is conveyed by tissue-specific mechanisms, at least for some genes.

Our RNA-FISH experiments confirmed that Tmem29 escapes XCI, adding yet another gene to the 15 or so escapees previously known in mouse [34]. Tmem29 escaped inactivation in $12 \%$ of the fibroblasts counted, while $K d m 5 c$ presented biallelic expression in $25 \%$ of the cells. This deviation suggests dissimilarity in control mechanisms for biallelism for these two genes, but we cannot fully rule out that differences in probe composition contributed to the divergence observed.

Genes escaping X-inactivation [35] and even the $\mathrm{Xi}$ heterochromatin itself [36] may underlie significant phenotypic differences between the sexes. It should however be pointed out that female mice that are monosomic for $\mathrm{X}$ are fertile and show a mild phenotype compared to women with Turner Syndrome [37].

\section{Small female-biased gene clusters on $\mathrm{X}$}

Our current investigation extended our previous discovery of female-biased gene clusters on the mouse $\mathrm{X}$-chromosome [22]. Here we investigate in more detail the cluster including $K d m 5 c$, because it contains the largest number of female-biased non-coding genes. Xinactive repressive histone marks, such as histone variant macroH2A1 (macroH2A1) and tri-methylation at lysine 27 of histone 3 (H3K27me3), are known to be depleted in escape domains [23,24]. It was previously shown by us and others that $K d m 5 c$ and D930009K15Rik are located in a chromosomal region virtually depleted of these repressive marks, and that both escape XCI [22-25]. Novel in the current study is the identification of three additional female-biased non-coding genes in this cluster, located downstream of $K d m 5 c$ and D930009K15Rik, apparently in the transition region of macroH2A1 and H3K27me3 enrichment (Figure 6A). This pattern is consistent with a model for the $K d m 5 c$ escape domain, in which $K d m 5 c$ and $D 930009 K 15 R i k$ are located in a transcriptionally active chromosomal domain while the subsequent noncoding genes are located in the transition region between active and silenced domains (Figure 6B). We note that one of these non-coding genes, 2900056M20Rik, was in a previous study classified as $\mathrm{X}$ inactivated [38], perhaps indicating tissue variability in escape among genes in the transition region. The coding genes located upstream (Iqsec2) and downstream (Tspyl2) of the female-biased $K d m 5 c$ cluster have previously been shown to be $\mathrm{X}$-inactivated [39], and therefore the limits of the $K d m 5 c$ escape domain are established. Whether the non-coding RNAs encoded in this escaping domain serve any function in the regulation of escape, the preservation of the boundary between active/inactive domains, or the silencing of neighbouring inactive genes, remains to be explored.

\section{Other mechanisms that may explain sex-biased expression of $\mathrm{X}$-encoded genes}

Escape from XCI is not the only mechanism that leads to female-biased expression of X-linked genes. For example, some of the female-biased genes may be controlled by regulators acting in trans, such as hormonally regulated factors. If this is so, the abundance of femalebiased genes on $\mathrm{X}$ may correlate with an overrepresentation of the corresponding transcription factor binding sites in X-gene promoters. Conversely, it is conceivable that $\mathrm{X}$-gene transcriptional down-regulating factors are enriched in males, and binding motifs for these factors are then similarly expected to be enriched on the X. Sex-bias of some of the identified genes may also reflect sex differences in cell composition within the tissues. Another possible mechanism for sex-biased gene expression is genomic imprinting of $\mathrm{X}$-chromosome genes $[41,42]$. Indeed, widespread sex-specific parent-of-origindependent allelic expression was reported recently in the brain of rodents in several studies [43-46]. This may also mediate or resolve sexually antagonistic effects [47]. If any of the mechanisms described above would affect one sex but not the other, the dosage compensation achieved by random $\mathrm{X}$-inactivation would be altered, resulting in sex-biased expression in the particular tissue in which the mechanism operates.

\section{Female-biased X-genes are evolutionarily old}

Multiple studies have demonstrated that different evolutionary processes and patterns are observed on $\mathrm{X}$-chromosome and autosomal chromosomes [48]. For example, a recent study showed that two bursts of gene gain on the X-chromosome occurred during the evolution of mammals [28]. Moreover, it was suggested that the X-chromosome recently acquired a burst of young male-biased genes (operating in testis), which is consistent with a fixation of recessive male-beneficial alleles by sexual antagonism [28]. Our results provide a different story in terms of the evolution of sex-biased genes acting in somatic tissues. While both studies indicate that female-biased genes are old, the results for male-biased genes differ between our study of somatic tissues and the study of gonadal expression. We found a moderately significant abundance of evolutionarily old genes also among male-biased genes, at least in the kidney. These combined results demonstrate that considerations on the potential effect of sexual antagonism during the evolution of X-chromosome will require a concerted analysis of the selective forces operating both in gonads and in somatic tissues. 


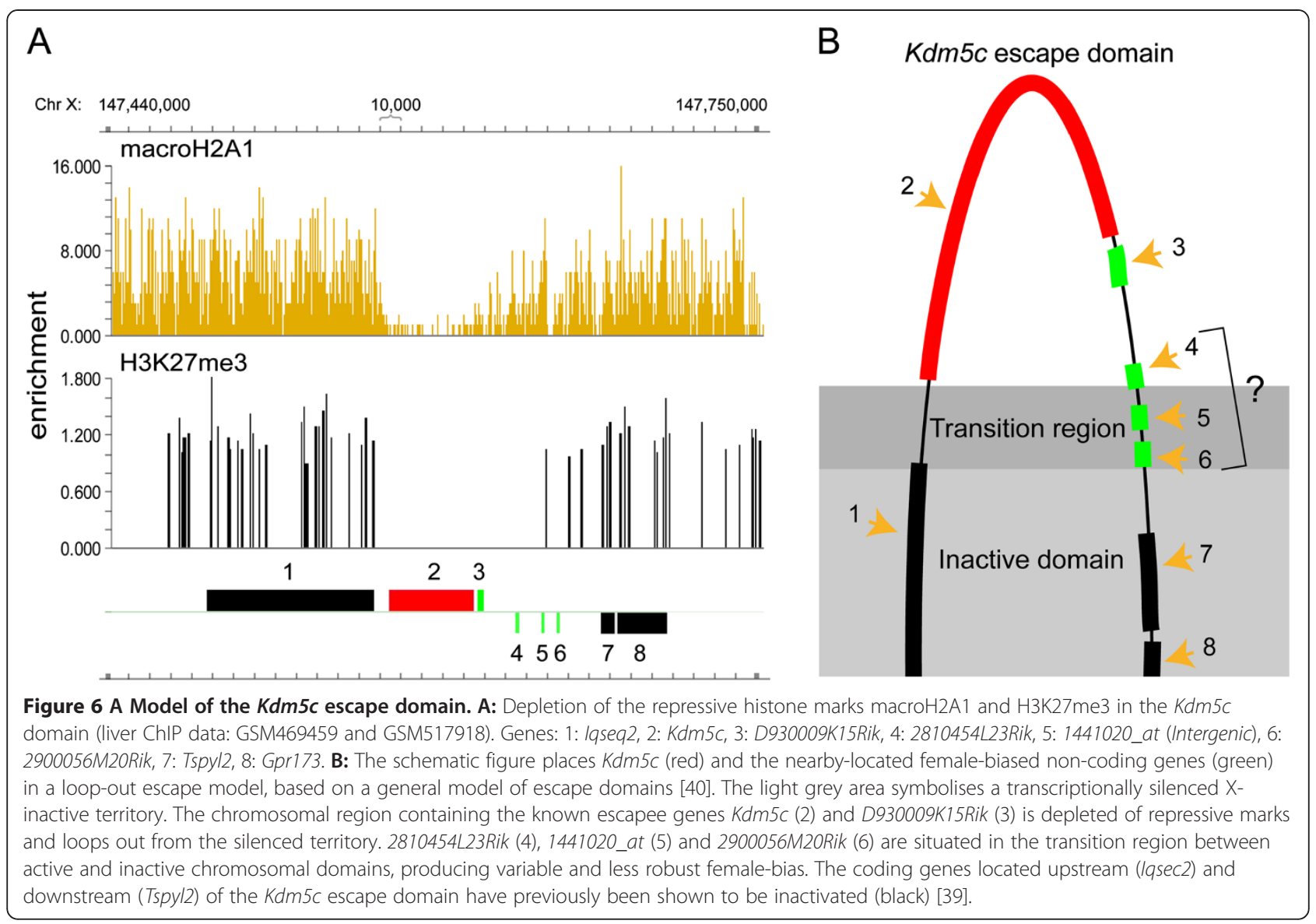

\section{Conclusions}

We performed a high-resolution study of sex-biased RNA expression of genes located on the mouse $\mathrm{X}$-chromosome in somatic tissues. We found that the mouse X-chromosome is significantly depleted of malebiased genes and enriched with female-biased genes. Our results in mice correlate with previous analyses of evolutionarily unrelated X-chromosomes in distant species such as Drosophila melanogaster and Caenohabditis elegans, indicating the generality of at least some of the selective forces acting on X-chromosomes. The expression of previously known genes that escape X-chromosome inactivation could explain only a minor part of the observed overrepresentation of female-biased genes in our data set, indicating either the existence of many tissue-specific escapees or the existence of additional sexskewed gene regulatory mechanisms operating on the Xchromosome. Among the newly identified female-biased genes, using RNA-FISH, the gene Tmem 29 was shown to escape X-inactivation in a small proportion of female fibroblasts interrogated. An analysis of gene age showed that evolutionarily old genes, pre-dating the radiation of placental mammals, are more frequently female-biased than younger genes. A similar pattern was observed among male-biased genes in kidney, contrasting with previously shown recent gain on the X-chromosome of male-biased genes expressed in testis.

\section{Methods}

\section{Data analysis}

We used array data (Affymetrix Mouse 430v.2) from GeneNetwork (University of Tennessee), available at: http://www.genenetwork.org. Datasets: Kidney: "Mouse kidney M340v2 Male (Aug06) RMA" (GN240), "Mouse kidney M340v2 Female (Aug06) RMA" (GN239); Liver: "GenEx BXD Sal Liver Affy M430 2.0 (Feb11) RMA Males" (GN311), "GenEx BXD Sal Liver Affy M430 2.0 (Feb11) RMA Females" (GN312); Lung: "HZI LungM430v2 (Apr08) RMA" (GN160); Striatum: "HBP Rosen Striatum M340V2 (Apr05) RMA" (GN69); Eye: "Hamilton Eye Institute Mouse Eye M430v2 Data Set (Sept08) RMA" (GN207); Hippocampus: "Hippocampus Consortium M340v2 (Jun06) RMA" (GN110). The number of biological specimens was 2-6 individuals per array, as specified for each dataset in the GeneNetwork database. The arrays were normalised with Robust Multichip Average (RMA), and intensities were log-transformed and standardised to a mean of 8 and a standard deviation of 2 . 
Differentially expressed (DE) genes were identified in each tissue using the Wilcoxon Mann-Whitney test (R v2.6.2 Environment for Statistical Computing http:// www.r-project.org, wilcox.test(); standard approximation of $\mathrm{p}$-values) at $\mathrm{p}<0.001$ or $\mathrm{p}<0.01$ (not adjusted for multiple testing), as described in the results section. Nonparametric (Wilcoxon) and parametric (t-test) tests showed similar results in number of DE probes. Significant DE X-linked probes using Wilcoxon (or t-test) were as follows for each tissue. Kidney: 287 (290), Liver: 176 (142), Lung: 48 (45), Striatum: 38 (28), Eye: 38 (35), Hippocampus: 25 (25). The Wilcoxon test was chosen to avoid violations of the assumption of normal distribution of probe intensities in each gene. The results for all probes (using both statistics) are available in Additional file 1 and Additional file 2. Since body weight and hormonal state were not controlled, the regulatory origin of any differential expression may be the compound effects of chromosomal constitution, hormonal differences and metabolic states between the sexes. The selection criterion for probes in Table 2was a consistent (defined as a significant deviation in the same direction) female-bias or male-bias $(\mathrm{p}<0.01)$ in more than one tissue. SignalMap v1.9.0.03 (NimbleGen) was used to visualise the distribution of sex-biased genes shown in Figure 2. The statistical significance of the observed over-/underrepresentation of chromosomal location for sex-biased genes was determined with a two-sided Fisher's exact test and the significance criterion $\mathrm{p}<0.01$. For the analysis shown in Figure $3 \mathrm{~B}\left(\mathrm{X}^{\prime}\right)$, we excluded the following known escapees: Xist, Shroom4, Mid1, Kdm6a, Kdm5c, Jpx, Ftx, Eif2s3x, Ddx3x, Car5b, Bgn, BC022960, D930009K15Rik, 6720401G13Rik, 5530601H04Rik, 2610029G23Rik, and 1810030007Rik [22-25,49,50]. The female mouse liver H3K27me3 [23] and macroH2A1 [24] data used in Figure 6 were downloaded from the Gene Expression Omnibus (http://www.ncbi.nlm.nih.gov/geo, Accession: GSE20617: GSM517918, GSE18963: GSM469459), and SignalMap v1.9.0.03 (NimbleGen) was used to visualize enrichment. For the gene age analysis, branch assignment of sex-biased genes was set according to Zhang et al. (Additional file 2) [28], matched by Ensembl ID, and the phylogeny in the upper section of Figure 5 was depicted accordingly (Zhang et al. 2010, page 2). The lower part of Figure 5 shows only those branches that were represented by at least 10 probes on the Mouse 430v.2 array. An apparent decrease in the proportion of sexbiased genes was observed around the split between branch 5 and 6. A two-sided Fisher's exact test was applied to determine the statistical significance of the differences in the proportion of sex-biased genes assigned to branches before or after this split. GO analysis was performed in DAVID Bioinformatics Resources 6.7, NIAID/NIH [26,27], using two reference sets: all X-chromosome genes in the
Mouse 430v.2 array, or all genes included in the array, independent of chromosomal location, as specified in Additional file 3 A-E.

\section{RNA-FISH}

Mouse embryonic fibroblasts (MEFs) derived from skin were cultured in Minimum Essential Medium Eagle (Sigma) supplemented with $2 \%$ L-glutamine, $10 \%$ fetal bovine serum and $1.5 \%$ penicillin/streptomycin (Invitrogen). Upon reaching confluence, MEFs were subcultured 1:4 in Culturewell MultiWell cell culture system (Molecular Probes) and grown until reaching 80\% confluence. Cultures were maintained at $37^{\circ} \mathrm{C}$ and $5 \% \mathrm{CO}_{2}$. Cells were fixed by $3 \%$ paraformaldehyde for $15 \mathrm{~min}$ at room temperature, followed by permeabilization in $0.5 \%$ TritonX-100 in PBS with $10 \mathrm{mM}$ Ribonucleoside Vanadyl Complex (New England Biolabs) for $5 \mathrm{~min}$. Fixed cells were stored in $70 \%$ ethanol at $-20^{\circ} \mathrm{C}$ until usage. Probes (Xist: RP23-84A16, Tmem29: RP23-84G23, Tmem164: RP23-239E14, Kdm5c: RP23-202H16, Ctps2: RP23-106K4, Rbm41: RP23-381C12, 4732460I02Rik/ Rbmx: RP23-44G23) were labeled with ChromaTide Alexa Fluor 488-5-dUTP (Molecular Probes) or Cy3dCTP (Amersham) PA53021, using the BioPrime Array CGH Genomic Labeling system (Invitrogen). Hybridisation with labelled DNA (40 ng/ $\mu \mathrm{l})$ and mouse Cot1 DNA (100 ng/ $\mu \mathrm{l})$ (Invitrogen) was performed overnight at $38^{\circ} \mathrm{C}$ in $2 \times \mathrm{SSC}, 50 \%$ formamide and $12 \%$ dextran sulfate, with $10 \mathrm{mM}$ Ribonucleoside Vanadyl Complex. Cells were washed with $2 \times$ SSC and $50 \%$ formamide ( $3 \times 5$ min; $\left.40^{\circ} \mathrm{C}\right)$ and $2 \times \operatorname{SSC}\left(3 \times 5 \mathrm{~min} ; 40^{\circ} \mathrm{C}\right)$. Slides were mounted with Vectashield (Vector Labs). Cell imaging was carried out using a confocal microscope (Zeiss LSM 510 META, Göttingen, Germany). The images presented in Figure 4 were uniformly processed and merged in Adobe Photoshop CS3 (Adobe).

\section{Additional files}

Additional file 1: Microarray results: genes on the X-chromosome.
The table shows the statistics from the sex-specific analysis for probes
mapping to the X-chromosome. "Biotype" is given according to Ensembl
(BioMart:Ensembl Genes 62/NCBIM37), "Branch" according to Zhang et al.
[28]. The colour code indicates the following significance: $p<0.01$; red:
female-biased, blue: male-biased. This level of significance was marked to
facilitate interpretation of the table, but note that the significance
criterion used in the main study was $p<0.001$.

Additional file 2: Microarray results: all chromosomal locations. The table shows the statistics from the sex-specific analysis for all probes present on the Affymetrix mouse 430v.2 array. The colour code indicates the following significance: $p<0.01$; red: female-biased, blue: male-biased. This level of significance was marked to facilitate interpretation of the table, but note that the significance criterion used in the main study was $p<0.001$.

Additional file 3: A-E. Gene Ontology analysis. Results from a Gene Ontology analysis of female- and male-biased X-linked and autosomal genes, using DAVID Bioinformatics Resources 6.7, NIAID/NIH [26,27]. The 
$\mathrm{GO}$ analysis was performed using as reference either $\mathrm{X}$-genes or all genes included in the microarray, as specified in the title of each table.

\section{Competing interests}

The authors declare that they have no competing interests.

\section{Authors' contributions}

Conceived the study: BR, EJ. Prepared the microarray data: RWW. Analysed the data: BR. Participated in the RNA-FISH experiments: BR, MMJ, KJR, GKP CK. Wrote the paper: BR, EJ, EHM, RS. All authors read and approved the final manuscript.

\section{Public data}

Gene Expression Omnibus (http://www.ncbi.nlm.nih.gov/geo, Accession: GSM469459, GSM517918)

GeneNetwork (http://www.genenetwork.org, Accession: GN69, GN110,

GN160, GN207, GN239, GN240, GN311, GN312)

\section{Acknowledgements}

We would like to express our gratitude towards Robert J. Rooney for the early access to the mouse liver data sets, and we are grateful to Susana Chuva De Sousa Lopes for her insightful comments on the manuscript. We acknowledge NIH NIAAA INIA Consortium for support of GeneNetwork. This project was funded by a grant from the Marcus Borgström Foundation to BR and EJ.

\section{Author details}

'Department of Organismal Biology, Evolutionary Biology Centre, Uppsala University, Uppsala, Sweden. ${ }^{2}$ Ludwig Institute for Cancer Research, Stockholm, Sweden. ${ }^{3}$ Department of Cell and Molecular Biology, Karolinska Institutet, Stockholm, Sweden. ${ }^{4}$ School of Life Sciences, University of Sussex, Brighton, United Kingdom. ${ }^{5}$ Department of Genetics and Pathology, Rudbeck Laboratory, Uppsala University, Uppsala, Sweden. ${ }^{6}$ Department of Medical and Clinical Genetics, Department of Biomedicine, The Sahlgrenska Academy, Gothenburg University, Gothenburg, Sweden. ${ }^{7}$ Department of Anatomy and Neurobiology, University of Tennessee, Knoxville, USA

Received: 23 December 2011 Accepted: 8 October 2012

Published: 10 November 2012

\section{References}

1. Mank JE: Sex chromosomes and the evolution of sexual dimorphism: lessons from the genome. Am Nat 2009, 173(2):141-150.

2. Lande R: Sexual Dimorphism, Seexual Selection, and Adaptation in Polygenic Characters. Evolution 1980, 34(2):292-305.

3. Albert AY, Otto SP: Sexual selection can resolve sex-linked sexual antagonism. Science 2005, 310(5745):119-121.

4. Gurbich TA, Bachtrog D: Gene content evolution on the X chromosome. Curr Opin Genet Dev 2008, 18(6):493-498.

5. Innocenti P, Morrow EH: The sexually antagonistic genes of Drosophila melanogaster. PLoS Biol 2010, 8(3):e1000335

6. Connallon T, Clark AG: The resolution of sexual antagonism by gene duplication. Genetics 2011, 187(3):919-937.

7. Bachtrog D: A dynamic view of sex chromosome evolution. Curr Opin Genet Dev 2006, 16(6):578-585.

8. Reinke V: Sex and the genome. Nat Genet 2004, 36(6):548-549.

9. Rice WR: Sex chromosomes and the evolution of sexual dimorphism. Evolution 1984, 38:735-742.

10. Parisi M, Nuttall R, Naiman D, Bouffard G, Malley J, Andrews J, Eastman S, Oliver B: Paucity of genes on the Drosophila X chromosome showing male-biased expression. Science 2003, 299(5607):697-700.

11. Reinke V, Smith HE, Nance J, Wang J, Van Doren C, Begley R, Jones SJ, Davis $\mathrm{EB}$, Scherer S, Ward S, et al: A global profile of germline gene expression in C. elegans. Mol Cell 2000, 6(3):605-616.

12. Yang $X$, Schadt EE, Wang S, Wang H, Arnold AP, Ingram-Drake L, Drake TA Lusis AJ: Tissue-specific expression and regulation of sexually dimorphic genes in mice. Genome Res 2006, 16(8):995-1004.
13. Lin H, Gupta V, Vermilyea MD, Falciani F, Lee JT, O'Neill LP, Turner BM: Dosage compensation in the mouse balances up-regulation and silencing of X-linked genes. PLoS Biol 2007, 5(12):e326.

14. Nguyen DK, Disteche CM: Dosage compensation of the active $X$ chromosome in mammals. Nat Genet 2006, 38(1):47-53.

15. Lyon MF: Gene action in the X-chromosome of the mouse (Mus musculus L.). Nature 1961, 190:372-373.

16. Fry JD: The genomic location of sexually antagonistic variation: some cautionary comments. Evolution 2010, 64(5):1510-1516.

17. Shapiro LJ, Mohandas T, Weiss R, Romeo G: Non-inactivation of an x-chromosome locus in man. Science 1979, 204(4398):1224-1226.

18. Migeon BR: Some insights into $X$ chromosome inactivation from studies of human cells. Ann Endocrinol (Paris) 1980, 41(4):275-280.

19. Goldman MA, Stokes KR, Idzerda RL, McKnight GS, Hammer RE, Brinster RL, Gartler SM: A chicken transferrin gene in transgenic mice escapes X-chromosome inactivation. Science 1987, 236(4801):593-595.

20. Brown CJ, Willard HF: Localization of a gene that escapes inactivation to the $\mathrm{X}$ chromosome proximal short arm: implications for $\mathrm{X}$ inactivation. Am J Hum Genet 1990, 46(2):273-279.

21. Mank JE: The $W, X, Y$ and $Z$ of sex-chromosome dosage compensation. Trends Genet 2009, 25(5):226-233.

22. Reinius B, Shi C, Hengshuo L, Sandhu KS, Radomska KJ, Rosen GD, Lu L, Kullander K, Williams RW, Jazin E: Female-biased expression of long noncoding RNAs in domains that escape X-inactivation in mouse. BMC Genomics 2010, 11:614

23. Yang F, Babak T, Shendure J, Disteche CM: Global survey of escape from X inactivation by RNA-sequencing in mouse. Genome Res 2010, 20(5):614-622.

24. Changolkar LN, Singh G, Cui K, Berletch JB, Zhao K, Disteche CM, Pehrson JR: Genome-wide distribution of $\mathrm{macroH} 2 \mathrm{~A} 1$ histone variants in mouse liver chromatin. Mol Cell Biol 2010, 30(23):5473-5483.

25. Lopes AM, Arnold-Croop SE, Amorim A, Carrel L: Clustered transcripts that escape X inactivation at mouse XqD. Mamm Genome 2011.

26. da Huang W, Sherman BT, Lempicki RA: Systematic and integrative analysis of large gene lists using DAVID bioinformatics resources. Nat Protoc 2009, 4(1):44-57

27. da Huang W, Sherman BT, Lempicki RA: Bioinformatics enrichment tools: paths toward the comprehensive functional analysis of large gene lists. Nucleic Acids Res 2009, 37(1):1-13.

28. Zhang YE, Vibranovski MD, Landback P, Marais GA, Long M: Chromosomal redistribution of male-biased genes in mammalian evolution with two bursts of gene gain on the X chromosome. PLoS Biol 2010, 8(10):e1000494.

29. Khil PP, Smirnova NA, Romanienko PJ, Camerini-Otero RD: The mouse $X$ chromosome is enriched for sex-biased genes not subject to selection by meiotic sex chromosome inactivation. Nat Genet 2004, 36(6):642-646.

30. Wang PJ, McCarrey JR, Yang F, Page DC: An abundance of X-linked genes expressed in spermatogonia. Nat Genet 2001, 27(4):422-426.

31. Parsch J: X chromosome: expression and escape. PLoS Genet 2009, 5(11):e1000724.

32. Jiang M, Ryu J, Kiraly M, Duke K, Reinke V, Kim SK: Genome-wide analysis of developmental and sex-regulated gene expression profiles in Caenorhabditis elegans. Proc Natl Acad Sci USA 2001, 98(1):218-223.

33. Thoemke K, Yi W, Ross JM, Kim S, Reinke V, Zarkower D: Genome-wide analysis of sex-enriched gene expression during $C$. elegans larval development. Dev Biol 2005, 284(2):500-508.

34. Yang C, Chapman AG, Kelsey AD, Minks J, Cotton AM, Brown CJ: Xchromosome inactivation: molecular mechanisms from the human perspective. Hum Genet 2011, 130:175-85.

35. Disteche CM: Escape from $\mathrm{X}$ inactivation in human and mouse. Trends Genet 1995, 11(1):17-22.

36. Wijchers PJ, Yandim C, Panousopoulou E, Ahmad M, Harker N, Saveliev A, Burgoyne PS, Festenstein R: Sexual dimorphism in mammalian autosomal gene regulation is determined not only by Sry but by sex chromosome complement as well. Dev Cell 2010, 19(3):477-484

37. Burgoyne PS, Baker TG: Oocyte depletion in XO mice and their XX sibs from 12 to 200 days post partum. J Reprod Fertil 1981, 61(1):207-212.

38. Tsuchiya KD Greally JM, Yi Y Noel KP. Truong JP, Disteche CM: Comparative sequence and $\mathrm{x}$-inactivation analyses of a domain of escape in human xp11.2 and the conserved segment in mouse. Genome Res 2004, 14(7):1275-1284

39. Li N Carrel L: Escape from $\mathrm{X}$ chromosome inactivation is an intrinsic property 
of the Jarid1c locus. Proc Natl Acad Sci USA 2008, 105(44):17055-17060.

40. Berletch JB, Yang F, Xu J, Carrel L, Disteche CM: Genes that escape from X inactivation. Hum Genet 2011, 130(2):237-245.

41. Skuse DH, James RS, Bishop DV, Coppin B, Dalton P, Aamodt-Leeper G, Bacarese-Hamilton M, Creswell C, McGurk R, Jacobs PA: Evidence from Turner's syndrome of an imprinted X-linked locus affecting cognitive function. Nature 1997, 387(6634):705-708.

42. Skuse DH: Genomic imprinting of the $X$ chromosome: a novel mechanism for the evolution of sexual dimorphism. J Lab Clin Med 1999, 133(1):23-32.

43. Wang X, Sun Q, McGrath SD, Mardis ER, Soloway PD, Clark AG: Transcriptome-wide identification of novel imprinted genes in neonatal mouse brain. PLoS One 2008, 3(12):e3839.

44. Gregg C, Zhang J, Weissbourd B, Luo S, Schroth GP, Haig D, Dulac C: Highresolution analysis of parent-of-origin allelic expression in the mouse brain. Science 2010, 329(5992):643-648.

45. Wang X, Soloway PD, Clark AG: Paternally biased X inactivation in mouse neonatal brain. Genome Biol 2010, 11(7):R79

46. Gregg C, Zhang J, Butler JE, Haig D, Dulac C: Sex-specific parent-of-origin allelic expression in the mouse brain. Science 2010, 329(5992):682-685.

47. Bonduriansky R, Chenoweth SF: Intralocus sexual conflict. Trends Ecol Evol 2009, 24(5):280-288.

48. Vicoso B, Charlesworth B: Evolution on the $X$ chromosome: unusual patterns and processes. Nat Rev Genet 2006, 7(8):645-653.

49. Tian D, Sun S, Lee JT: The long noncoding RNA, Jpx, is a molecular switch for X chromosome inactivation. Cell 2010, 143(3):390-403.

50. Chureau C, Chantalat S, Romito A, Galvani A, Duret L, Avner P, Rougeulle C: Ftx is a non-coding RNA which affects Xist expression and chromatin structure within the X-inactivation center region. Hum Mol Genet 2011, 20(4):705-718.

doi:10.1186/1471-2164-13-607

Cite this article as: Reinius et al:: Abundance of female-biased and paucity of male-biased somatically expressed genes on the mouse X-chromosome. BMC Genomics 2012 13:607.

\section{Submit your next manuscript to BioMed Central and take full advantage of:}

- Convenient online submission

- Thorough peer review

- No space constraints or color figure charges

- Immediate publication on acceptance

- Inclusion in PubMed, CAS, Scopus and Google Scholar

- Research which is freely available for redistribution 